\title{
Mid-infrared spectrometer and camera for the Origins Space Telescope
}

\author{
Itsuki Sakon $\odot$, a,* Thomas L. Roellig, ${ }^{\mathrm{b}}$ Kimberly Ennico-Smith $\odot,{ }^{\mathrm{b}}$ \\ Taro Matsuo, ${ }^{\text {c }}$ Yuji Ikeda, ${ }^{\text {d }}$ Tomoyasu Yamamuro, ${ }^{e}$ Keigo Enya, \\ Takehiko Wada, ${ }^{f}$ Mitsunobu Kawada, ${ }^{f}$ Aoi Takahashi $\odot,{ }^{g}$ Yuki Sarugaku, \\ Naofumi Fujishiro, ${ }^{i}$ Naoshi Murakami, ${ }^{j}$ Jun Nishikawa, ${ }^{\text {g Takayuki Kotani, }}$ \\ Shohei Goda, ${ }^{k}$ Masayuki Ido, ${ }^{k}$ Satoshi Itoh, ${ }^{k}$ Takahiro Tsuboi, \\ Takahiro Sumi, ${ }^{k}$ Masatsugu Kamiura $\odot{ }^{1}$, Takeo Manome, ${ }^{1}$ Naoto Iida, \\ Kentaro Yanagibashi, ${ }^{1}$ Thomas Greene $\odot{ }^{\text {b }}$ Bernard's Helvensteijn, ${ }^{b}$

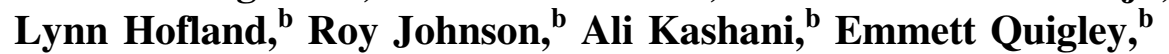 \\ Robert McMurray, ${ }^{b}$ Hanae Inami $\odot,{ }^{m}$ Denis Burgarella $\odot,{ }^{n}$ and \\ the Origins Space Telescope mission concept study team ${ }^{\dagger}$ \\ ${ }^{a}$ University of Tokyo, Graduate Schools of Science, Department of Astronomy, Tokyo, Japan \\ ${ }^{b}$ NASA Ames Research Center, Space Sciences Division, Moffett Field, California, \\ United States \\ 'Nagoya University, Graduate School of Science, Department of Physics, Nagoya, Japan \\ ${ }^{\mathrm{d}}$ Photocoding, Kyoto, Japan \\ ${ }^{\text {e} O p t o c r a f t, ~ 3-16-8-101, ~ H i g a s h i ~ H a s h i m o t o, ~ S a g a m i h a r a, ~ J a p a n ~}$ \\ ${ }^{\mathrm{f}}$ Institute of Space and Astronautical Science, Japan Aerospace Exploration Agency, \\ Sagamihara, Japan \\ ${ }^{\mathrm{g}}$ National Astronomical Observatory of Japan, Tokyo, Japan

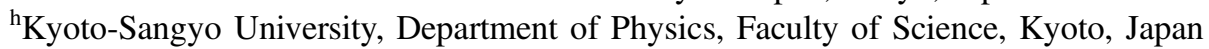 \\ ${ }^{\mathrm{i}}$ Teikyo University, General Medical Education and Research Center, Tokyo, Japan \\ ${ }^{j}$ Hokkaido University, Division of Applied Physics, Faculty of Engineering, Hokkaido, Japan \\ ${ }^{\mathrm{k}}$ Osaka University, Department of Earth and Space Science, Osaka, Japan \\ ${ }^{1}$ Kyocera Corp., Corporate Fine Ceramics Group, Kyoto, Japan \\ ${ }^{\mathrm{m}}$ Hiroshima University, Hiroshima Astrophysical Science Center, Hiroshima, Japan \\ ${ }^{\mathrm{n}}$ Aix-Marseille University, Laboratoire d'Astrophysique de Marseille, Marseille Cedex 13, \\ France
}

\begin{abstract}
The mid-infrared spectrometer and camera transit spectrometer (MISC-T) is one of the three baseline instruments for Origins Space Telescope (Origins) and provides the capability to assess the habitability of nearby exoplanets and search for signs of life. MISC-T employs a densified pupil optical design, and $\mathrm{HgCdTe}$ and Si:As detector arrays. This optical design allows the instrument to be relatively insensitive to minor line-of-sight pointing drifts and telescope aberrations, and the detectors do not require a sub-Kelvin refrigerator. MISC-T has three science spectral channels that share the same field-of-view by means of beam splitters, and all channels are operated simultaneously to cover the full spectral range from 2.8 to $20 \mu \mathrm{m}$ at once with exquisite stability and precision $(<5 \mathrm{ppm}$ between 2.8 to $11 \mu \mathrm{m},<20 \mathrm{ppm}$ between 11 and $20 \mu \mathrm{m}$ ). A Lyot-coronagraph-based tip-tilt sensor located in the instrument fore-optics uses the light reflected by a field stop, which corresponds to $0.3 \%$ of the light from the target, to send fine pointing information to the field steering mirror in the Origins telescope. An additional MISC Wide Field Imager (WFI) is studied as an upscope option for the Origins. MISC-WFI offers a wide field imaging $\left(3^{\prime} \times 3^{\prime}\right)$ and low-resolution spectroscopic capability with filters and gratingprisms (grisms) covering 5 to $28 \mu \mathrm{m}$. The imaging capability of the MISC-WFI will be used for general science objectives. The low-resolution spectroscopic capability in MISC-WFI with a resolving power $\mathrm{R}(=\lambda / \Delta \lambda)$ of a few hundreds will be used to measure the mid-infrared dust features and ionic lines at $z$ up to $\sim 1$ in the Origins mission's Rise of Metals and Black Hole
\end{abstract}

\footnotetext{
*Address all correspondence to Itsuki Sakon, isakon@astron.s.u-tokyo.ac.jp

${ }^{\dagger}$ Members of the study team are listed in the Origins Space Telescope Mission Concept Study Report, which is available at https://asd.gsfc .nasa.gov/firs/docs/
} 
Feedback programs. The MISC-WFI also serves as a focal plane pointing and guiding instrument for the observatory, including when the MISC-T channel is performing its exoplanet spectroscopy observations. (C) 2021 Society of Photo-Optical Instrumentation Engineers (SPIE) [DOI: 10 .1117/1.JATIS.7.1.011013]

Keywords: astronomy; mid-infrared; transit spectroscopy.

Paper 20088SS received Jun. 16, 2020; accepted for publication Jan. 27, 2021; published online Mar. 11, 2021.

\section{Introduction}

The Origins Space Telescope (Origins) traces our cosmic history, from the formation of the first galaxies and the rise of metals to the development of habitable worlds and present-day life. Origins does this through exquisite sensitivity to infrared emission and absorption features associated with ions, atoms, molecules, dust, water vapor and ice, and observations of extra-solar planetary atmospheres and protoplanetary disks. Origins operates in the wavelength range from 2.8 to $588 \mu \mathrm{m}$ and is more than 1000 times more sensitive than its predecessors due to its large, cold $(4.5 \mathrm{~K})$ telescope and advanced instruments.

The mid-infrared spectrometer and camera (MISC) instrument studied for the Origins had two major components: a transit spectrometer (MISC transit spectrometer; hereafter MISC-T) and a camera with low-resolution grating-prism (grism) spectroscopy (MISC Wide Field Imager; hereafter MISC-WFI). The latter component became an upscope option during the team's descope process. MISC-T can be flown as a stand-alone instrument and acquisition and guiding would be provided by the observatory. MISC-WFI could be built, if the observatory requires it, to provide wide-field imaging for science at wavelengths $<30 \mu \mathrm{m}$ and acquisition and guiding for the observatory. The design of MISC-WFI would include the tip-tilt (TT) requirements for MISC-T. The baseline concept of the MISC instrument is described in Sec. 2, and the upscope concept of the full MISC instrument in Sec. 3.

\section{MISC Baseline}

MISC-T is one of the three baseline instruments for the Origins. ${ }^{1}$ The parameters of the baseline instrument design and the estimated performance of MISC-T is shown in Table 1.

MISC-T provides spectroscopy simultaneously over 2.8 to $20 \mu \mathrm{m}$ with exquisite stability and precision of $<5 \mathrm{ppm}$ (1 sigma) from 2.8 to $10 \mu \mathrm{m}$ with $R=50$ to 100 and $<20 \mathrm{ppm}$ ( 1 sigma) from 10 to $20 \mu \mathrm{m}$ with $R \sim 200$. An additional MISC spectrometer and camera is studied and described in Sec. 3 as an upscope option. However, for historical reasons, ${ }^{2}$ MISC remains as the instrument acronym.

\subsection{MISC Transit Spectrometer SCIENCE Traceability}

The science drivers for MISC-T are captured by the exoplanet case, Are we alone? $?^{3,4}$

The three main science objectives for the Origins' science goal of Do planets orbiting M-dwarf stars support life? are to determine (1) what fraction of terrestrial M- and K-dwarf planets have tenuous, clear, or cloudy atmosphere, (2) what fraction of terrestrial M-dwarf planets are temperate, and (3) what types of temperate, terrestrial M-dwarf planets support life. The MISC-T is optimized, in particular, for the measurements of bio-signatures in the atmospheres of transiting exoplanets. The first step will be to use the entire mid-IR wavelength range of the MISC-T to search for and find at least 10 exoplanets with surface temperatures suitable for liquid water. Step 2 will make deeper observations of these candidates at 2.8 to $10 \mu \mathrm{m}$ to find at least four candidates with bio-indicator molecules $\left(\mathrm{CO}_{2}\right.$ and $\left.\mathrm{H}_{2} \mathrm{O}\right)$. Finally, these candidates will be observed for longer integration times over multiple transits to detect the presence of

$2329-4124 / 2021 / \$ 28.00$ (C) 2021 SPIE 
Table 1 The MISC-T instrument fact sheet.

\begin{tabular}{|c|c|}
\hline Parameter & MISC-T \\
\hline Observing modes & АОT01: MISC ultra stable spectroscopy \\
\hline Spectral range & $\begin{array}{l}2.8 \text { to } 20 \mu \mathrm{m} \text { (MISC-T-S; } 2.8 \text { to } 5.5 \mu \mathrm{m} \text {, MISC-T-M; } 5.5 \text { to } 11 \mu \mathrm{m} \text {, MISC-T-L; } \\
11 \text { to } 20 \mu \mathrm{m} \text { ) }\end{array}$ \\
\hline Resolving power & $\begin{array}{l}R=50 \text { to } 100 \text { in } 2.8 \text { to } 5.5 \mu \mathrm{m} \text { (MISC-T-S) } \\
R=50 \text { to } 100 \text { in } 5.5 \text { to } 11 \mu \mathrm{m} \text { (MISC-T-M) } \\
R=165 \text { to } 295 \text { in } 11 \text { to } 20 \mu \mathrm{m} \text { (MISC-T-L) }\end{array}$ \\
\hline Angular resolution & Cannot attain spatially resolved information within the field of view \\
\hline Field of view & $\begin{array}{l}3.0^{\prime \prime} \text { in radius (MISC-T-S) } \\
3.0^{\prime \prime} \text { in radius (MISC-T-M) } \\
2.0^{\prime \prime} \text { in radius (MISC-T-L) }\end{array}$ \\
\hline Detectors & $\begin{array}{l}\text { A } 2 \mathrm{k} \times 2 \mathrm{k} \mathrm{HgCdTe} \text { detector array }(30 \mathrm{~K}) \text { for MISC-T-S } \\
\text { A } 2 \mathrm{k} \times 2 \mathrm{k} \mathrm{HgCdTe} \text { detector array }(30 \mathrm{~K}) \text { for MISC-T-M } \\
\text { A } 2 \mathrm{k} \times 2 \mathrm{k} \mathrm{Si} \text { As detector array with a calibration source for MISC-T-L } \\
\text { A } 256 \times 256 \mathrm{HgCdTe} \text { detector array }(30 \mathrm{~K}) \text { for MISC-T-TTS }\end{array}$ \\
\hline Stability $^{a}$ & $\begin{array}{l}3.19 \mathrm{ppm} \text { at } 3 \mu \mathrm{m}(R=50) \\
3.37 \mathrm{ppm} \text { at } 5 \mu \mathrm{m}(R=50) \\
3.84 \mathrm{ppm} \text { at } 8 \mu \mathrm{m}(R=50) \\
5.51 \mathrm{ppm} \text { at } 10 \mu \mathrm{m}(R=50) \\
8.63 \mathrm{ppm} / 9.31 \mathrm{ppm} \text { at } 14 \mu \mathrm{m}(R=50 \text {; Glasse model/Wright model, } I=0 \mathrm{deg}) \\
14.76 \mathrm{ppm} / 16.33 \mathrm{ppm} \text { at } 14 \mu \mathrm{m}(R=200 \text {; Glasse model/Wright model, } I=0 \mathrm{deg}) \\
13.11 \mathrm{ppm} / 16.04 \mathrm{ppm} \text { at } 20 \mu \mathrm{m}(R=50 \text {; Glasse model/Wright model, } I=0 \mathrm{deg}) \\
29.31 \mathrm{ppm} / 37.04 \mathrm{ppm} \text { at } 20 \mu \mathrm{m}(R=300 \text {; Glasse model/Wright model, } I=0 \mathrm{deg})\end{array}$ \\
\hline $\begin{array}{l}\text { Sensitivity; } \\
\text { SNR/sqrt(hr) }\end{array}$ & $\begin{array}{l}\text { SNR/sqrt(hr })=12952 \text { at } 3.3 \mu \mathrm{m} \\
\text { SNR/sqrt(hr) }=9873 \text { at } 6.3 \mu \mathrm{m} \\
\text { SNR/sqrt(hr) }=4570 \text { at } 14 \mu \mathrm{m}\end{array}$ \\
\hline Saturation Limit ${ }^{\mathrm{c}}$ & $\begin{array}{l}29.8 \mathrm{Jy} \text { at } 3.3 \mu \mathrm{m} \\
27.5 \mathrm{Jy} \text { at } 6.3 \mu \mathrm{m} \\
4.4 \mathrm{Jy} \text { at } 14 \mu \mathrm{m}\end{array}$ \\
\hline
\end{tabular}

${ }^{a}$ On timescales of a few hours assuming 85 transits for a $K \sim 8$ mag M-type star

${ }^{\mathrm{b}}$ Assuming a $R=50$ with a $10.8 \mathrm{~K}$-mag star

${ }^{\mathrm{C}} \mathrm{Calculated}$ for the shortest readout time; assuming partial readout, $10 \mu \mathrm{s}$ per pixel per read, two reads per pixel to sample up the ramp

bio-signature molecules $\left(\mathrm{H}_{2} \mathrm{O}, \mathrm{CO}_{2}, \mathrm{CH}_{4}, \mathrm{O}_{3}\right.$, and $\left.\mathrm{N}_{2} \mathrm{O}\right)$ to at least the $3.6 \sigma$ level. This requires a capability to measure the transmission spectra sensitive to $\mathrm{CH}_{4}(3.3,7.6 \mu \mathrm{m}), \mathrm{N}_{2} \mathrm{O}(4.6,7.8$ and $17 \mu \mathrm{m}), \mathrm{O}_{3}(9.6 \mu \mathrm{m}), \mathrm{H}_{2} \mathrm{O}(6.3 \mu \mathrm{m})$, and $\mathrm{CO}_{2}(4.2 \mu \mathrm{m})$ in the wavelength range from 3 to $19 \mu \mathrm{m}$. The MISC-T provides the Origins with the observational capabilities to achieve the Origins' exoplanet objectives. 


\subsection{MISC-T Instrument Description}

\subsubsection{MISC-T operation principle}

The MISC-T module is primarily dedicated to pointed observations to detect bio-signatures in habitable worlds in primary and secondary exoplanet transits. MISC-T has three channels (T-S, T-M, and T-L) that share the same field-of-view (FOV) by means of beam splitters, and all channels are operated simultaneously to cover the full spectral range from 2.8 to $20 \mu \mathrm{m}$ at once. The science traceability matrix requirements for the spectral accuracy for exoplanet transit spectroscopy are $<5 \mathrm{ppm}$ from 2.8 to $10 \mu \mathrm{m}$ and $<20 \mathrm{ppm}$ from 10 to $20 \mu \mathrm{m}$. To reach this performance requires system optimization involving not only the MISC-T optical design and detector/readout electronics choices, but also observatory performance issues such as the pointing performance and the nature and variability of the Origins telescope aberrations. Various instrument design concepts were considered during the present study. The instrument design will be finalized in Origins Phase A. For proof-of-concept and costing purposes, we designed MISC-T with a novel densified pupil optical design, and HgCdTe and Si:As detector arrays. The densified pupil design divides up the light from a point-source in the instrument pupil plane up into a number of individual spectrometer optical channels and is described in more detail in Ref. 5. This design is relatively insensitive to minor line-of-sight pointing drifts and telescope aberrations, and the detectors do not require a sub-Kelvin refrigerator. It does have the disadvantage of reduced sensitivity to faint sources compared to the traditional spectrometer design since the point source spectra are spread out over a relatively large number of detector pixels. However, for this science program with the relatively bright host star targets, spectro-photometric stability is more important than faint-source sensitivity.

To demonstrate the potential of this novel optical design, a testbed system has been constructed using the densified pupil design and a $1024 \times 1024$ Si:As Impurity Band Conduction array. Performance testing has been ongoing at NASA Ames Research Center and so far, the design has met all of its design requirements.

It is noteworthy that an alternative instrument design concept was considered in which transition edge sensor (TES) detectors were fed by a more conventional spectrometer, with Winston cones used to concentrate more photons to improve sensitivity at these short wavelengths. As this design concept has not been analyzed as thoroughly for overall system performance, especially with respect to errors introduced by telescope image quality and pointing variation, it was not chosen as the baseline, but further work on this alternate concept will be carried into the Phase A Concept Study.

Densified pupil spectroscopy is a new optical system approach for transit spectroscopy ${ }^{5,6}$ that will greatly improve spectro-photometric performance in the presence of optical disturbances. With pupil densification, the MISC-T science image will not be disturbed by modest telescope pointing jitter (e.g., 10 mas) or telescope aberrations. The large number of science pixels minimizes the effects of intra- and inter-pixel sensitivity variations. Reference pixels also provide potential detector baseline common-mode gain fluctuation calibration using a cold photon shield mask. As part of the Origins technology development plan, the team will refine an optimal pixelto-pixel calibration technique using an ultra-stable internal calibration source (see Sec. 2.2.3.) that meets with the instrument requirement of $<20 \mathrm{ppm}$ at $\lambda=10.5$ to $20 \mu \mathrm{m}$. The MISC-T block diagram is shown in Fig. 1.

No simultaneous operation between MISC-T and other instruments is expected; therefore, the high operating temperature for the $\mathrm{HgCdTe}$ detectors will not be a source of noise to the other instruments.

A Lyot-coronagraph-based tip-tilt sensor (TTS) located in the instrument fore-optics uses the light reflected by a field stop, which corresponds to $0.3 \%$ of the light from the target, to send fine pointing information to the fine steering mirror (FSM) in the telescope optical train. ${ }^{7}$ The TTS measures the long-term drift using the point source image formed on the focal plane through a Lyot stop and feeds this information back to the observatory to mitigate the longterm drift down to 2 mas over a timescale of a few hours. 


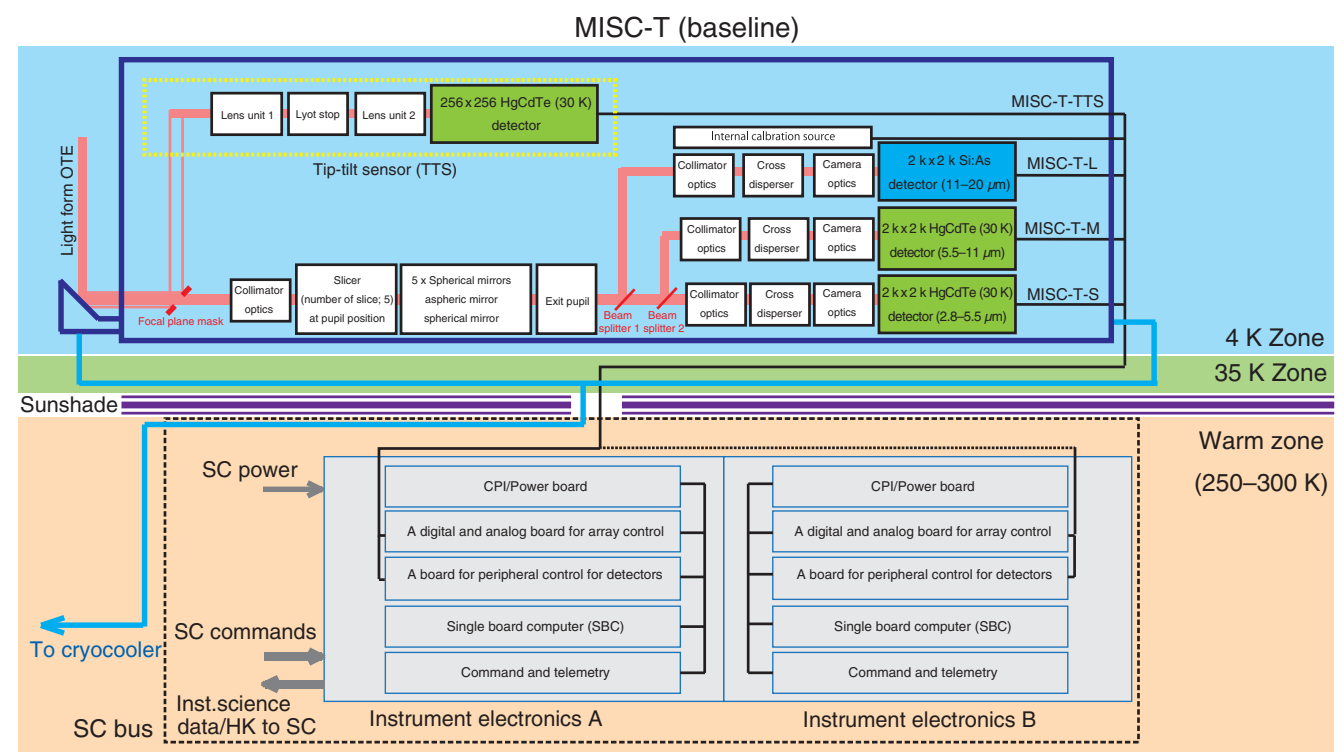

Fig. 1 The block diagram of the MISC-T.

\subsubsection{MISC-T optical design}

The MISC-T optical design is based on the densified pupil spectroscopy. ${ }^{5}$ Conventional spectrographs (e.g., long slit) use focal plane division: light from multiple positions in the focal plane is dispersed into multiple spectra. In contrast, the densified pupil spectrograph uses pupil-plane division and produces spectra from multiple sub-apertures of the pupil plane. It should be emphasized that in spite of the novel arrangement of optics in the densified pupil design, the optical components themselves are completely ordinary. The performance of this design is currently being demonstrated in tests at NASA Ames Research Center. The densified pupil spectrograph is composed of a pupil division/densification system followed by a normal spectrograph system (See Fig. 2).

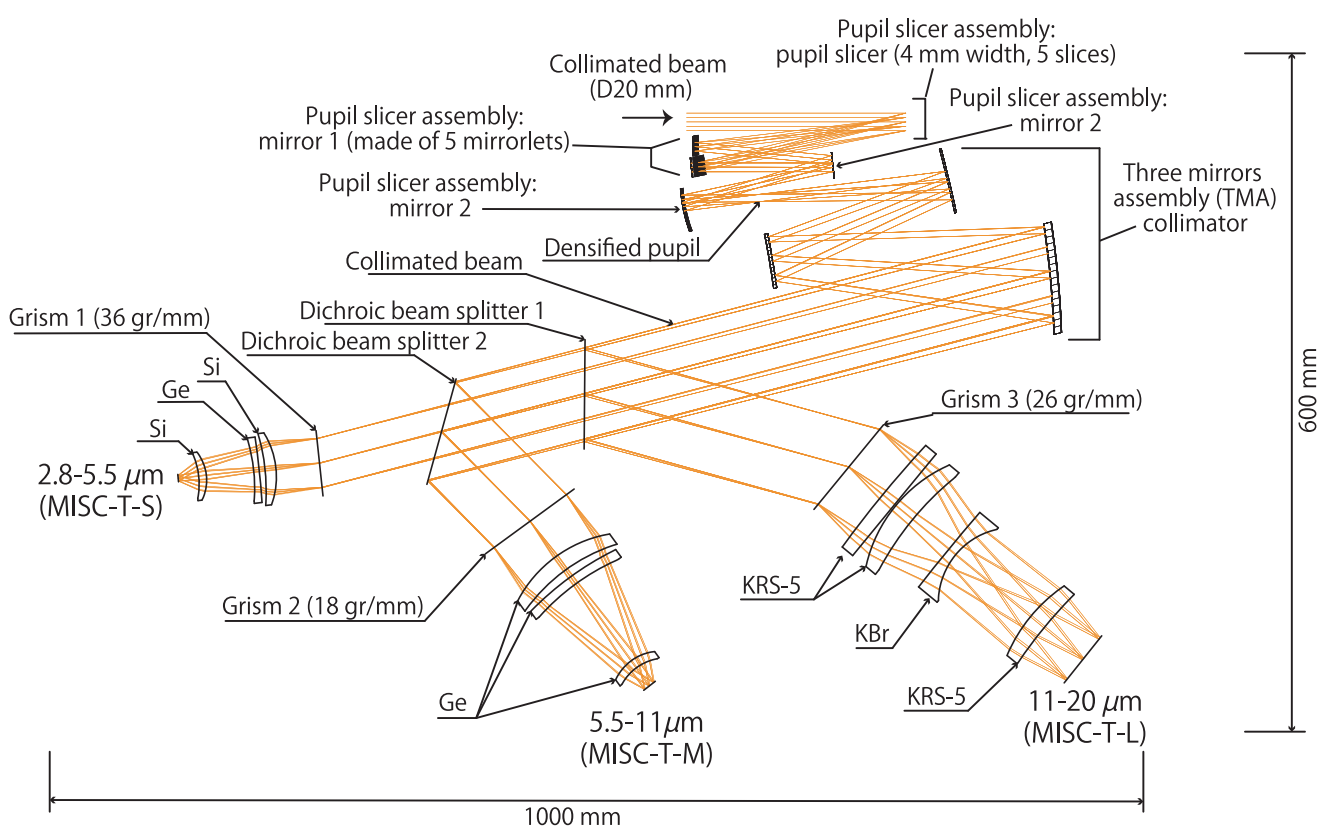

Fig. 2 The MISC-T Optical Design. The three spectrometers are fed by a collimated beam that emerges from the pupil slicer assembly and the TMA collimator. The dimensions of the instrument in $\mathrm{mm}$ are indicated. 

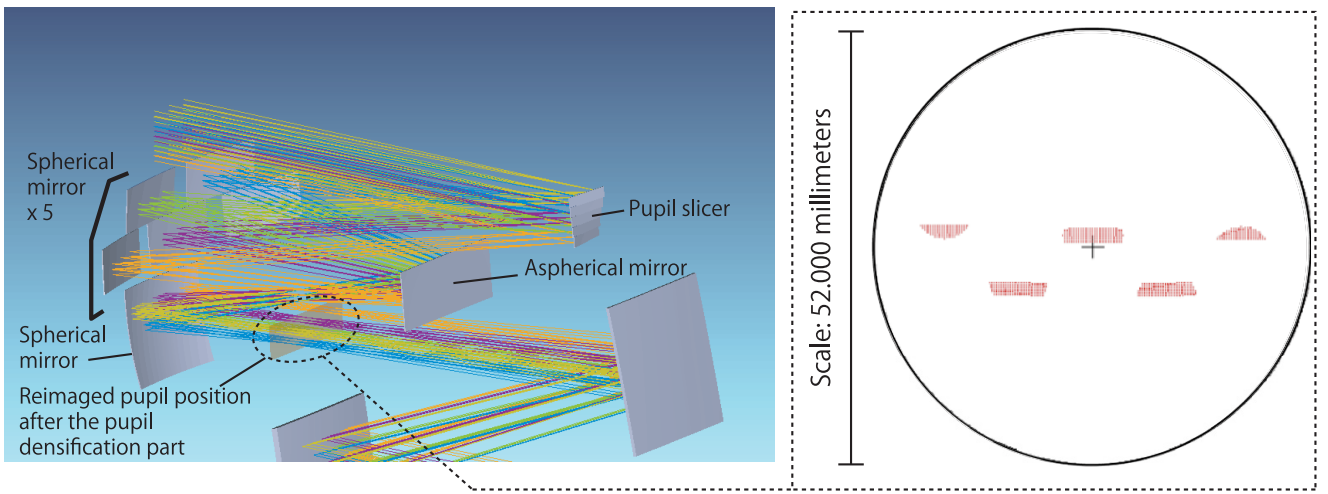

Fig. 3 The telescope pupil is reimaged and sliced as shown at left, with the slices separated as shown in the image on the right.

The pupil densification plays a key role in performing highly stable spectroscopy. Because of the pupil division into five slices in the case of MISC-T, and the densification of the divided pupil, each of the densified sub-pupils acts as a point source, and each corresponding beam is collimated by triple mirror assembly (TMA) collimator as shown in Fig. 2. The pupil is sliced into five segments, as shown in Fig. 3.

The first dichroic beam splitter reflects the light of 11 to $20 \mu \mathrm{m}$, which is led to the spectrograph of T-L, and transmits the light of $<11 \mu \mathrm{m}$. The second dichroic beam splitter reflects the light of 5.5 to $11 \mu \mathrm{m}$, which is led to the spectrograph of T-M, and transmits the light of $<5.5 \mu \mathrm{m}$, which is led to the spectrograph of T-S. Field stops with radii of 3." 0,3 . " 0 , and 2 . " 0 are installed in the spectrograph T-S, T-M, and T-L channels, respectively, to reduce the zodiacal background photons. The field stop aperture size determines the FOV size, and no spatially resolved information within the FOV is obtained.

Each spectrograph employs a transmission grism to disperse the light in one dimension as a function of the wavelength on a detector plane. Each of the five spectra shares 271 pixels in the dispersion direction by 27 pixels in the cross-dispersion direction for the T-S, 551 pixels in the dispersion direction by 54 pixels in the cross-dispersion direction for the T-M, and 1586 pixels in the dispersion direction by 65 pixels in the cross-dispersion direction for the T-L. The layout of these spectra onto the detector arrays is shown in Fig. 4. The linear dispersion $(\Delta \lambda)$ is set to 0.054, 0.108 , and $0.074 \mu \mathrm{m}$ for T-S, T-M, and T-L, respectively.

The TTS corrects any long-term drift in the telescope pointing. We conducted a trade study between a Lyot-Coronagraph-based field stop with a dedicated TTS array versus. a guider camera with a dichroic beam splitter to extract 2 to $2.8 \mu \mathrm{m}$ light of the target. The study showed a clear advantage in the Lyot-Coronagraph method in terms of MISC-T optical throughput, and in simplicity and cost. The TTS uses only $0.3 \%$ of the light from the target host star, which is reflected by the focal plane mask in the fore-optics, and the majority $(\sim 98 \%)$ of the light from

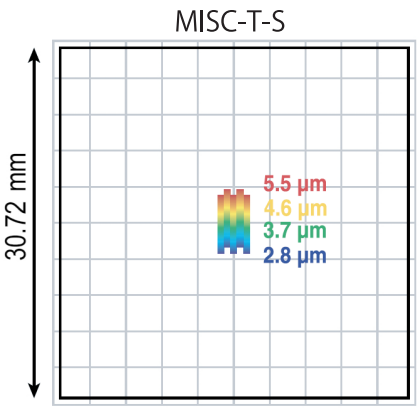

(a)

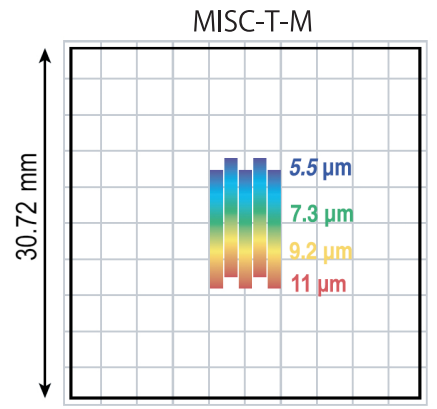

(b)

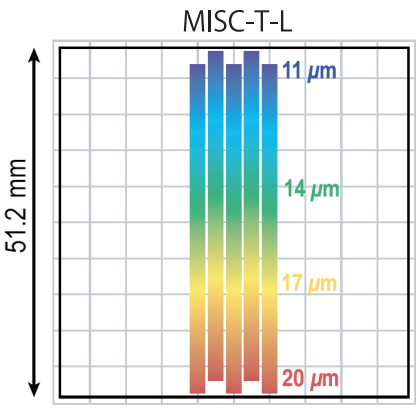

(c)

Fig. 4 The spectra from the five pupil slices are overlaid on the (a) short; (b) mid; and (c) long focal planes. 


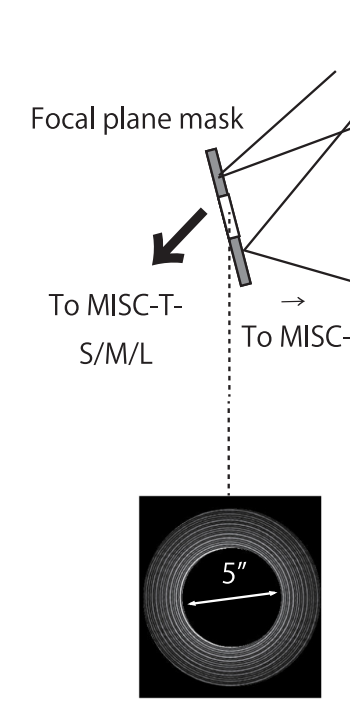

(a)

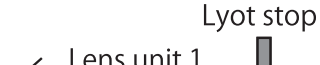

Lyot stop

(b)

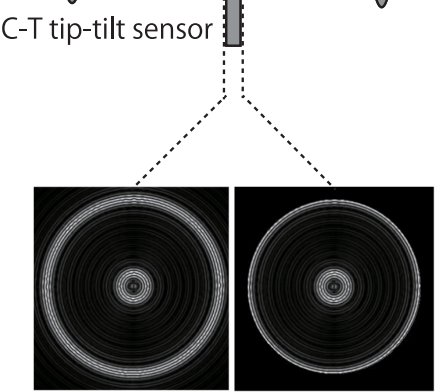

(c)
Lens unit 2 Detector plane

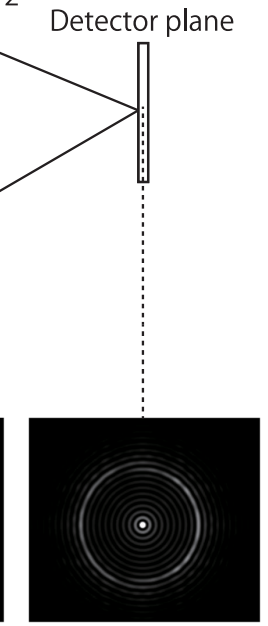

(d)

Fig. 5 A schematic view of the MISC-T TTS showing the results of a simulation of a PSF image (a) at the Lyot-Coronagraph focal plane mask; (b) just before the Lyot Stop; (c) just after the Lyot stop; and (d) at the detector plane.

the target passes through $\sim 5^{\prime \prime}$ aperture in the focal plane mask and is led to the T-S/M/L spectrometer channels. The long-term drift is measured by examining the position of the point-spread function (PSF) centroid of the image produced on the detector plane of the TTS through the Lyot stop. Assuming that the actual Lyot stop size is the same size as the Origins pupil and has an accuracy of $1 \%$, the TTS will work for targets of M-type stars brighter than $K=10 \mathrm{mag}$. The TTS feeds back this information to the observatory, and the long-term drift can be mitigated down to 2 mas for M-type star brighter than $K=10$ mag. Figure 5 shows the MISC-T TTS schematic.

\subsubsection{MISC-T detectors and read-out electronics}

The MISC-T's baseline employs two types of detectors: (1) HgCdTe that is bonded to a Si readout multiplexer, and (2) Si Blocked Impurity Band (BIB) design that is bonded to a Si readout multiplexer. ${ }^{8}$ Each of the MISC-T-S and T-M optical paths contains a $2 \mathrm{k} \times 2 \mathrm{k} \mathrm{HgCdTe}$ detector array operated at $30 \mathrm{~K}$, and the MISC-T TTS employs a $256 \times 256 \mathrm{HgCdTe}$ detector array operated at $\sim 30 \mathrm{~K}$. HgCdTe detectors developed for the Near Earth Object Survey Mission (NEOSM) have successfully demonstrated performance out to $10 \mu \mathrm{m} .{ }^{9}$ The MISC-T-L optical path contains a $2 \mathrm{k} \times 2 \mathrm{k} \mathrm{Si}$ :As detector array bonded to a Si readout multiplexer operating at $\sim 8 \mathrm{~K}$ to provide good detective quantum efficiency over the 10.5 to $20 \mu \mathrm{m}$ wavelength range. Ultra-stable internal calibration sources are employed to help achieve the required detector stability of $<20 \mathrm{ppm}$. These sources are composed of a combination of an infrared source black-body combined with a shorter-wavelength photocell detector used for temperature control. The photocell measures the source output in its blackbody Wien limit, allowing very sensitive feedback of the calibration source temperature, while the infrared wavelengths used for the infrared detector calibration are in the blackbody Rayleigh-Jeans limit. The black-body, whose light path comes from a region just outside the optical path coming from the telescope, is defocused and provides a flood illumination of the array.

Amplification of the signals from the detectors is a straightforward reuse of similar technology from previous space applications of $\mathrm{HgCdTe}$ and Si:As BIB detectors, including Spitzer, James Webb Space Telescope (JWST), Wide-field Infrared Space Explorer (WISE) and NEOSM. This method employs dedicated satellite chips located in close proximity to the detectors operating at cryogenic temperatures. The cable run from the cold satellite chips near the detectors to the warm electronics in the Origins design is shorter than it is in JWST, so the difficulties that were experienced with the long cable runs in the latter mission should be 
Table 2 The MISC observing modes (baseline) and data rates.

\begin{tabular}{lcc}
\hline \hline AOT & Mode & Data rate \\
\hline AOT01 & MISC Ultra Stable Spectroscopy & $1.59 \mathrm{Mbps}^{\left(\text {nominal }^{\mathrm{a}}\right)}-3.73 \mathrm{Mbps}\left(\mathrm{max}^{\mathrm{b}}\right)$ [MISC-T] \\
& $0.37 \mathrm{Mbps}^{\mathrm{c}}$ [MISC-T TTS] \\
\hline \hline
\end{tabular}

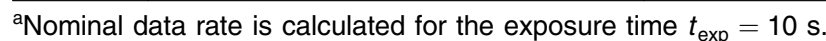

${ }^{\mathrm{b}} \mathrm{Max}$ data rate is calculated for the shortest exposure time $t_{\exp }=4 \mathrm{~s}$.

${ }^{\mathrm{C}}$ MISC-T TTS assumes to read and downlink $32 \times 32$ pixels around the PSF centroid at $20 \mathrm{~Hz}$.

much reduced. MISC-T incorporates flexible readout patterns and a variety of readout strategies including double-correlated and Fowler sampling techniques. Due to the extreme detector stability requirements, the readout electronics will need to be carefully designed and stabilized in temperature.

\subsubsection{MISC-T observing mode and data rates}

MISC-T has a single observing mode: AOT01 (Table 2). MISC-T-S, T-M, and T-L share the same FOV by means of beam splitters and are simultaneously operated to produce a complete 2.8 to $20 \mu \mathrm{m}$ spectrum at once.

\subsubsection{MISC-T thermal and mechanical design}

MISC-T instrument components are located in two thermal zones. The instrument optics and detectors are located in a cold zone at the same $\sim 4.5 \mathrm{~K}$ temperature as the telescope. The detectors have a weak thermal link to this cold temperature so they can be heated slightly and held at a stable operating temperature. This operating temperature is $\sim 30 \mathrm{~K}$ for the $\mathrm{HgCdTe}$ detectors and $\sim 7 \mathrm{~K}$ for the Si:As detector. Baffling in the cold instrument prevents IR light leakage from the warmer detectors from reaching the SI:As detector. This was successfully demonstrated by WISE, which had $32 \mathrm{~K}$ detectors and $8 \mathrm{~K}$ detectors located in the same instrument. The drive electronics that handle instrument data collection and communication with the spacecraft are located in the $\sim 300 \mathrm{~K}$ warm zone.

The MISC-T 3D solid model is shown in Fig. 6. To reduce MISC-T instrument mass, beryllium was assumed as a baseline material for the mirrors and mirror support structures, and the base plate. Cordierite CO-720, a ceramic material by Kyocera, has also been studied as an alternative material for the MISC-T and was used for the test instrument to demonstrate the performance of the densified pupil spectroscope at NASA Ames Research Center. Table 3 lists the mass breakdown of cold component estimated each for beryllium and Cordierite models. MISC-T does not have any moving parts or mechanisms, which is very important to achieve high spectro-photometric stability. Table 4 lists MISC-T resource requirements (volume, mass, and power).

\subsubsection{MISC-T instrument control}

The MISC-T instrument includes redundant dual-string warm electronics boxes (WEB A and WEB B for MISC-T). Each electronics box contains a single board computer (SBC) and three boards: (1) a CPI/ Power board, (2) a digital and analog board for array control (two $2 \times 2 \mathrm{k}$ $\mathrm{HgCdTe}$ arrays for T-S and T-M, one $2 \mathrm{k} \times 2 \mathrm{k} \mathrm{Si}$ :As array for T-L, one $256 \mathrm{k} \times 256 \mathrm{HgCdTe}$ array for T TTS), and (3) a board for peripheral control for detectors (two $2 \times 2 \mathrm{k} \mathrm{HgCdTe}$ arrays for T-S and T-M, one $2 \mathrm{k} \times 2 \mathrm{k}$ Si:As array for T-L, one $256 \times 256 \mathrm{HgCdTe}$ array for T TTS). The SBC calculates the centroid position of the PSF on the TTS detector array and sends this information back to the observatory. The FSM in the telescope optical $\operatorname{train}^{7}$ corrects for the pointing accuracy and mitigates the long-term drift down to a few mas over a timescale of a few hours. The detector biasing and readout electronics need to be very stable so that the detectors themselves can achieve their relative photometric stability requirement, which will necessitate careful temperature control of the electronics boxes.

J. Astron. Telesc. Instrum. Syst. $\quad$ 011013-8 Jan-Mar 2021 • Vol. 7(1) 


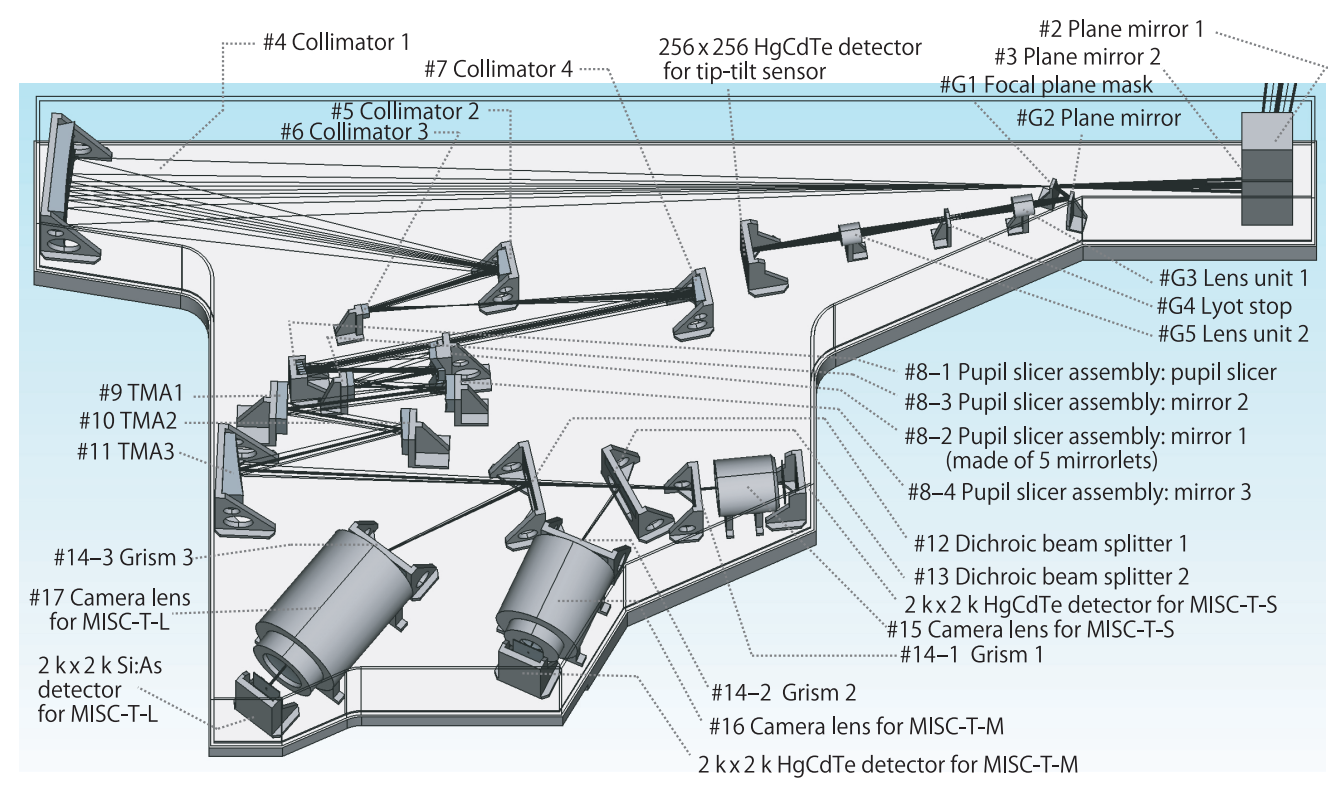

Fig. 6 A 3D solid model of the MISC-T instrument indicating the optical path of the light within the instrument and the location of the mirrors, optical elements and supporting structures on the optical baseplate.

\subsubsection{Risk management approach}

As an instrument on a NASA Class A mission, the MISC-T instrument has a fully redundant, dual-string, cross-strapped design. The MISC-T instrument has a warm electronics box each with two redundant sets of warm electronics, as shown in the block diagram (Fig. 1). Within an electronics box, the SBC and the rest of the warm electronics are cross-strapped as shown in Fig. 7. Although the focal plane arrays are not dual redundant, their multiplexer readouts are arranged so that sections of the arrays fail gracefully, as was done for the JWST instrument arrays.

\subsubsection{MISC-T alignment, integration, test, and calibration}

The MISC-T module will require its own unique facilities for testing and calibration at the instrument level before it is delivered for integration with the observatory. Aside from the normal vibration, acoustic, and electromagnetic interference (EMI) environmental testing, the MISCT module will need a thermal vacuum test chamber that can be cooled to liquid helium temperatures that is large enough to enclose the complete cold section of the MISC-T instrument and is IR light-tight enough that enough that stray light within the MISC-T detectors' sensitivity wavelengths is no greater than the infrared background that would be expected on orbit. This test chamber should allow the introduction of IR signals at the expected levels of the on-orbit target sources and the ability to verify the spectral response of the spectrometer. Finally, the test facility will require an ultra-stable light source that can verify the $\sim$ few ppm pixel-pixel detector stability requirements needed for the exoplanet transit measurements. This test facility will need to allow connections between the cold MISC-T instrument assembly in the test chamber and the warm MISC-T drive electronics over flight-like cables.

The warm MISC-T instrument drive electronics will not require any particular test facilities other than the usual test facilities used to conduct vibration, acoustic, EMI, and thermal vacuum tests. A spacecraft simulator will be required to test command and data transfers between the MISC-T and spacecraft electronics.

It will be necessary to develop specialized test hardware, but although the scope of this development will be significant, it is straightforward, with no new technology development required. MISC-T instrument I\&T is expected to take 12 months, assuming all the external test support hardware and software is ready in time by the start of testing. 
Table 3 Mass breakdown of MISC-T cold component.

\begin{tabular}{|c|c|c|c|}
\hline \multirow[b]{2}{*}{ Subassembly/part name } & & \multicolumn{2}{|c|}{ Mass $(\mathrm{kg})$} \\
\hline & & $\begin{array}{l}\text { Beryllium } \\
\text { model }^{\mathrm{a}}\end{array}$ & $\begin{array}{l}\text { CO720 } \\
\text { model }^{\mathrm{b}}\end{array}$ \\
\hline \multirow[t]{5}{*}{ TTS } & \#G1 Focal Plane Mask & 0.30 & 0.50 \\
\hline & \#G2 Plane Mirror & 0.30 & 0.50 \\
\hline & \#G3 Lens Unit 1 & 1.00 & 1.67 \\
\hline & \#G4 Lyot Stop & 0.30 & 0.50 \\
\hline & \#G5 Lens Unit 2 & 1.00 & 1.67 \\
\hline \multirow[t]{6}{*}{ Relay Optics and Mirror Supports } & \#2 Plane Mirror 1 & 0.30 & 0.50 \\
\hline & \#3 Plane Mirror 2 & 0.30 & 0.50 \\
\hline & \#4 Collimator 1 & 2.00 & 3.33 \\
\hline & \#5 Collimator 2 & 0.33 & 0.56 \\
\hline & \#6 Collimator 3 & 0.17 & 0.28 \\
\hline & \#7 Collimator 4 & 0.17 & 0.28 \\
\hline \multirow{7}{*}{$\begin{array}{l}\text { Pupil Slicer and Densification Optics } \\
\text { and Mirror Supports }\end{array}$} & \#8-1 Pupil Slicer Assembly: Pupil Slicer & 0.12 & 0.20 \\
\hline & $\begin{array}{l}\text { \#8-2 Pupil Slicer Assembly: Mirror } 1 \\
\text { (made of } 5 \text { mirrorlets) }\end{array}$ & 0.25 & 0.42 \\
\hline & \#8-3 Pupil Slicer Assembly: Mirror 2 & 0.15 & 0.25 \\
\hline & \#8-4 Pupil Slicer Assembly; Mirror 3 & 0.15 & 0.25 \\
\hline & \#9 TMA1 & 0.20 & 0.33 \\
\hline & \#10 TMA2 & 0.20 & 0.33 \\
\hline & \#11 ТМАЗ & 0.63 & 1.06 \\
\hline \multirow[t]{3}{*}{ MISC-T-S Optics and Mirror Supports } & \#12 Dichloic beam splitter 1 & 2.00 & 2.00 \\
\hline & \#14-1 Grism 1 & 1.00 & 1.00 \\
\hline & \#15 Camera Lens for MISC-T-S & 8.00 & 8.00 \\
\hline \multirow[t]{3}{*}{ MISC-T-M Optics and Mirror Supports } & \#13 Dichloic beam splitter 2 & 2.00 & 2.00 \\
\hline & \#14-2 Grism 2 & 1.00 & 1.00 \\
\hline & \#16 Camera Lens for MISC-T-M & 8.00 & 8.00 \\
\hline \multirow[t]{2}{*}{ MISC-T-L Optics and Mirror Supports } & \#14-3 Grism 3 & 1.00 & 1.00 \\
\hline & \#17 Camera Lens for MISC-T-L & 8.00 & 8.00 \\
\hline \multicolumn{2}{|l|}{ Instrument Optical Bench for MISC-T } & 20.00 & 34.00 \\
\hline \multicolumn{2}{|l|}{ CFRP Light Shield } & 4.00 & 4.00 \\
\hline \multicolumn{2}{|l|}{ Detector (MISC-T-S) and Detector Box } & 1.50 & 1.50 \\
\hline \multicolumn{2}{|l|}{ Detector (MISC-T-M) and Detector Box } & 1.50 & 1.50 \\
\hline \multicolumn{2}{|l|}{ Detector (MISC-T-L) and Detector Box } & 1.50 & 1.50 \\
\hline \multicolumn{2}{|l|}{ Detector (MISC-T TTS) and Detector Box } & 1.50 & 1.50 \\
\hline \multicolumn{2}{|l|}{ Total } & 68.87 & 88.13 \\
\hline
\end{tabular}

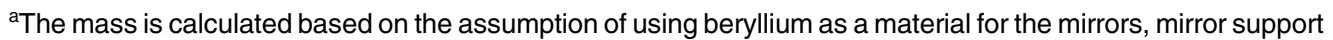
structures, and the base plate.

${ }^{\mathrm{b}}$ The mass is calculated based on the assumption of using Cordierite $\mathrm{CO} 220$ as a material for the mirrors, mirror support structures, and the base plate. 
Table 4 MISC-T instrument resource requirements.

\begin{tabular}{|c|c|c|c|c|c|}
\hline \multirow{2}{*}{$\begin{array}{l}\text { Instrument } \\
\text { resource type }\end{array}$} & \multicolumn{2}{|l|}{ Resource and units } & \multicolumn{2}{|c|}{ MISC-T (baseline) } & \multirow[b]{2}{*}{ Total } \\
\hline & Resource & Units & MISC-T & MISC-T TTS & \\
\hline $\begin{array}{l}\text { Information } \\
\text { related to the } \\
\text { mechanical } \\
\text { model }\end{array}$ & Volume (cold component) & $\begin{array}{c}\mathrm{m}^{3} \\
(\mathrm{~m} \times \mathrm{m} \times \mathrm{m})\end{array}$ & \multicolumn{2}{|c|}{$\begin{array}{l}0.15(2 \times 0.25 \times 0.20) \\
\text { Foreoptics }+ \text { TTS } \\
0.12(1 \times 0.8 \times 0.15) \\
\text { collimator + pupil } \\
\text { mapping and } \\
\text { densification Part + } \\
\text { Spectrographs }\end{array}$} & 0.27 \\
\hline \multirow{12}{*}{$\begin{array}{l}\text { Information } \\
\text { related to the } \\
\text { thermal model }\end{array}$} & Mass (cold component) & $\mathrm{kg}$ & \multicolumn{2}{|c|}{$68.87^{a}$} & 68.87 \\
\hline & Mass (warm component) & $\mathrm{kg}$ & \multicolumn{2}{|c|}{16.00} & 16.00 \\
\hline & Mass (harnessing and others) & $\mathrm{kg}$ & \multicolumn{2}{|c|}{16.36} & 16.36 \\
\hline & Total mass & $\mathrm{kg}$ & \multicolumn{2}{|c|}{101.23} & 101.23 \\
\hline & Total peak power (cold part) & W & 0.159 & 0.003 & 0.162 \\
\hline & Total peak power (warm part) & W & 10 & 3 & 13 \\
\hline & Total average power (cold part) & W & 0.009 & 0.003 & 0.012 \\
\hline & Total average power (warm part) & W & 10 & 3 & 13 \\
\hline & Total standby power (cold part) & W & 0.009 & 0.003 & 0.012 \\
\hline & Total standby power (warm part) & W & 10 & 3 & 13 \\
\hline & Average power dissipation (detectors) & W & 0.00804 & 0.00268 & 0.01072 \\
\hline & Average power dissipation (heater) & W & 0.009 & 0.003 & 0.012 \\
\hline
\end{tabular}

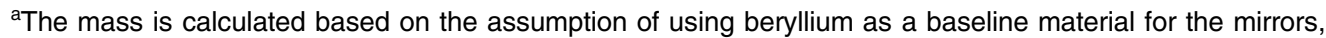
mirror support structures, and the base plate.

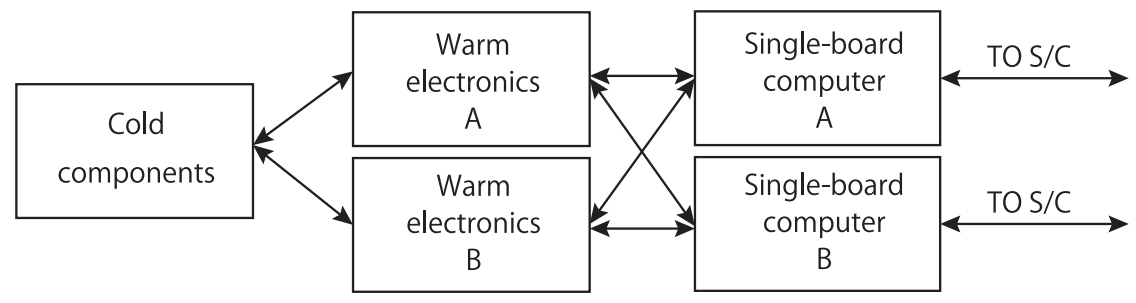

Fig. 7 The cross-strapping design used in each of the MISC-T modules' warm electronics provides dual-string fault tolerance.

\subsubsection{MISC-T predicted performance}

The MISC-T performance is calculated based on the following assumptions.

Throughput: The throughput curves as a function of the wavelength are calculated for T-S, $\mathrm{T}-\mathrm{M}$, and T-L, taking account of the reflectance of mirrors (four mirrors for telescope optics, three mirrors for fore-optics, three collimator mirrors, seven mirrors in the densified pupil spectrograph), the transmittance/reflectance of the dichroic beam splitters, grating efficiency, transmittance of the AR-coated lenses, detector quantum efficiency, and contamination/slit loss. The diffraction losses in the instrument were minimized with oversized optics (at least 12 $\lambda / D$ ). The final throughput including detector quantum efficiency is plotted in Fig. 8. 


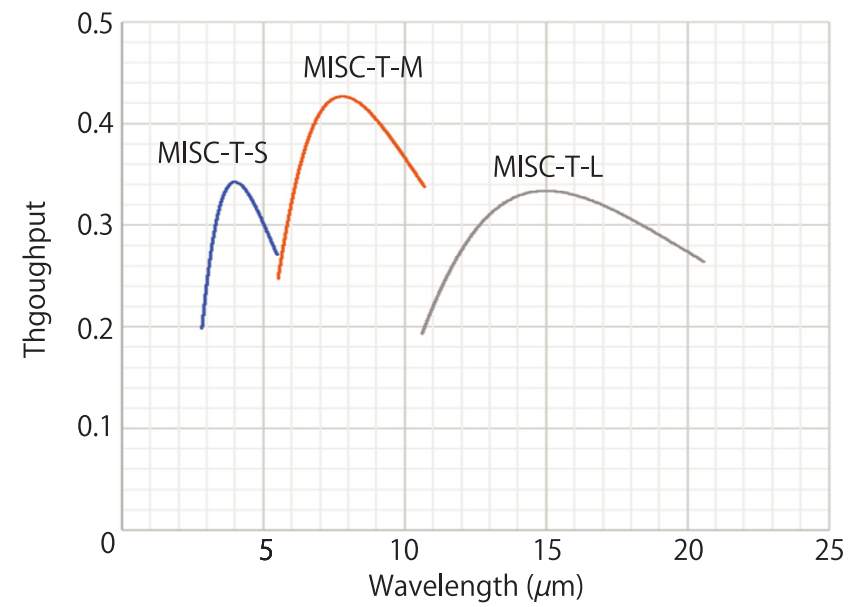

Fig. 8 The final throughput as a function of wavelength used for MISC-T performance calculations.

Zodiacal light: In addition to the light from the host star, there will be additional infrared light coming from the Zodiacal background. As the field stops have to be relatively large (3" radius for T-S and T-M, 2" radius for T-L) to capture the majority of the poorly focused, short-wavelength light from the $30 \mu \mathrm{m}$ diffraction-limited Origins telescope, the shot noise from this extra light can be a significant source of signal stability errors at some wavelengths. The Glasse Zodiacal model $^{10}$ is employed for the low background case outside the ecliptic plane while the Wright Zodiacal model for the ecliptic plane $(l=0)^{11}$ is employed for the high background case. The surface brightness of the zodiacal light, including scattered light and emissive components at representative wavelengths, is summarized in Table 5.

Pointing jitter and long-term drift: The major source of high-frequency pointing jitter is due to the cryocooler and is expected to be $<50$ mas, with a current best estimate of 9 mas RMS. Low frequency pointing drift has a much stronger effect on spectral stability. The LyotCoronagraph-based TT sensor (TTS) in the MISC-T instrument is used for measuring the long-term drift so that it can be compensated with the telescope FSM, if necessary. Together the performance is predicted to be 1mas drift over a timescale of a few hours.

Detector performance: A $2 \mathrm{k} \times 2 \mathrm{k} \mathrm{HgCdTe}$ detector array operated at $30 \mathrm{~K}$ is used in each of the MISC-T-S and T-M channels and a $2 \mathrm{k} \times 2 \mathrm{k}$ Si:As detector array operated at $\sim 8 \mathrm{~K}$ is used in MISC-T-L. The detector dark current is assumed to be $1 \mathrm{e}^{-} \mathrm{s}^{-1}$ pixel $^{-1}$ for the $2 \mathrm{k} \times 2 \mathrm{k} \mathrm{HgCdTe}$

Table 5 The surface brightness of the zodiacal light models used for the MISC-T performance calculations.

\begin{tabular}{lcc}
\hline \hline Wavelength $(\mu \mathrm{m})$ & $\begin{array}{c}\text { Low-background case } \\
\left(\mathrm{MJy} \mathrm{sr}^{-1}\right)\end{array}$ & $\begin{array}{c}\text { High-background case } \\
\left(\mathrm{MJy} \mathrm{sr}^{-1}\right)(\text { Wright et al. })\end{array}$ \\
\hline 3 & 0.1 & 0.92 \\
5 & 0.35 & \\
6 & 1 & 22.76 \\
8 & 3 & \\
10 & 8.5 & 46.58 \\
11 & 11 & 68.46 \\
14 & 14 & \\
20 & 17 & \\
\hline \hline
\end{tabular}




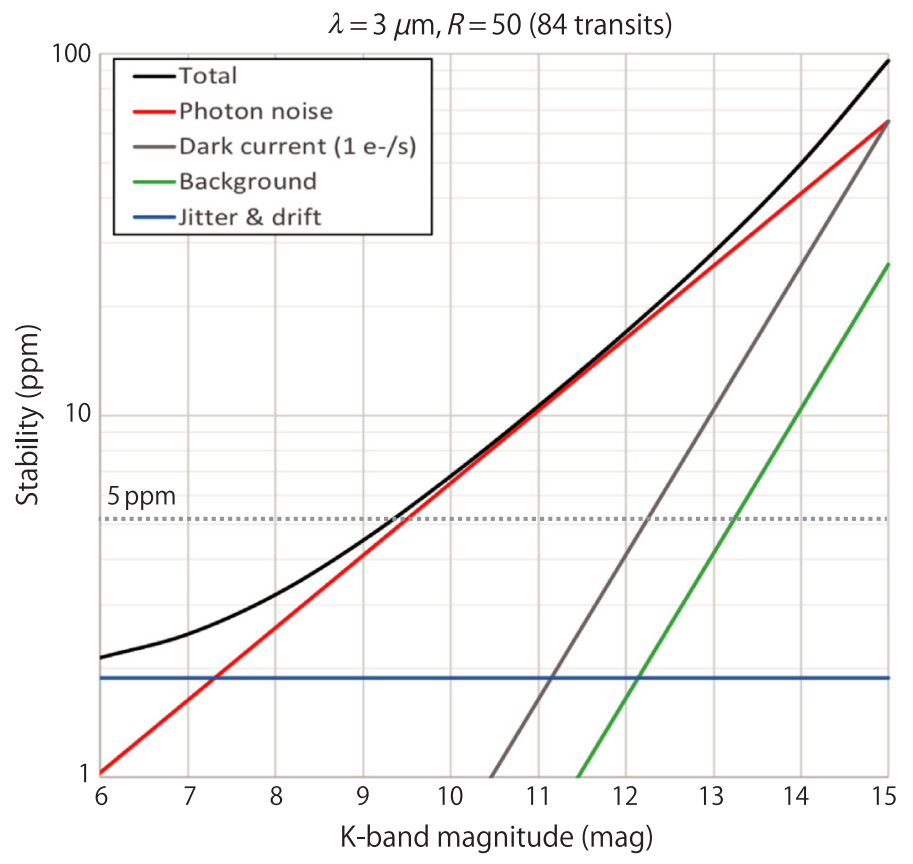

Fig. 9 The current best estimate of the spectro-photometric stability achieved by MISC-T at $3 \mu \mathrm{m}$ with $R=50$.

detector and $0.2 \mathrm{e}^{-} \mathrm{s}^{-1}$ pixel $^{-1}$ for the $2 \mathrm{k} \times 2 \mathrm{k} \mathrm{Si}$ :As detector. ${ }^{12}$ In addition to the science pixels, the reference and background pixels are applied for calibration of the systematic components present in the semiconductor device and ROIC. Since the time-variation component is common to all of the detector pixels, the common systematic components from the reference and background data are reconstructed and subtracted from the science data. The offset component estimated from the time- and pixel-averaged background data is also subtracted from the science data. This calibration procedures ${ }^{13}$ enable us to obtain the transit signal that is free from both the systematic and offset components. The dark current and background light are assumed to be constant during the transit observation. Thus, the stabilization of the detector's temperature and calibration of the dark current value using the time series of the detector temperature are required.

Based on these assumptions, the team simulated the light curves of late-M type stars at different K-band magnitudes and calculated the spectro-photometric accuracy at representative wavelengths for each type of late-M type star. The results calculated for 3, 5, 8, 10, 14, and $20 \mu \mathrm{m}$ assuming 84 transits and for a spectral resolving power of $R=50$ are shown in Figs 9-16. Noise sources due to photon shot noise, dark current, zodiacal light, jitter, and drift are included as shown. Aside from dark current, other detector and readout-electronics noise sources are not included. As an example, a bright star will have more integrated photons in 84 transits, so that the dark current noise contribution from the detectors will be relatively smaller. The flat contribution of the jitter and drift noise to the signal-to-noise over different host star brightnesses may seem odd at first, but in fact this term is a constant that is independent of the stellar magnitude.

\section{MISC Upscope}

The MISC instrument upscope configuration consists of a second separate module, the MISC WFI module, in addition to the MISC-T. The parameters of the baseline instrument design and the estimated performance of MISC WFI module are shown in Table 6.

The MISC WFI offers a wide field imaging $\left(3^{\prime} \times 3^{\prime}\right)$ and low-resolution spectroscopic capability with filters and grisms for 5 to $28 \mu \mathrm{m}$. As needed, the MISC WFI is also used for focal plane guidance for itself and MISC-T and the other Origins science instruments. Since the 


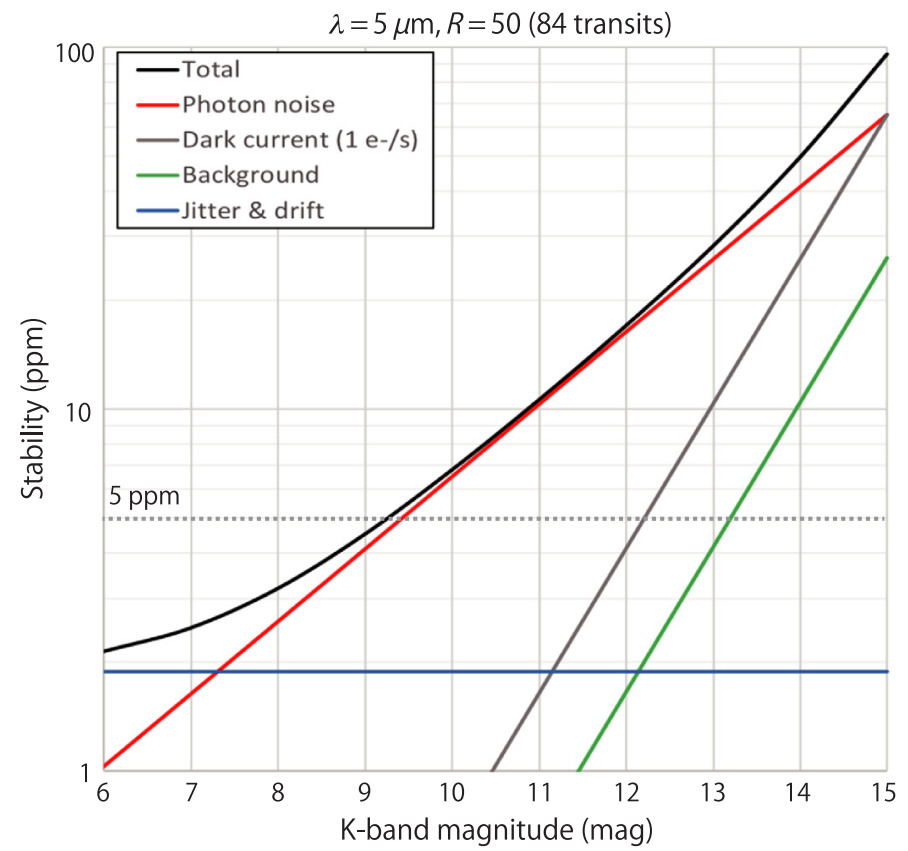

Fig. 10 Same as Fig. 9 but for at $5 \mu \mathrm{m}$ with $R=50$.

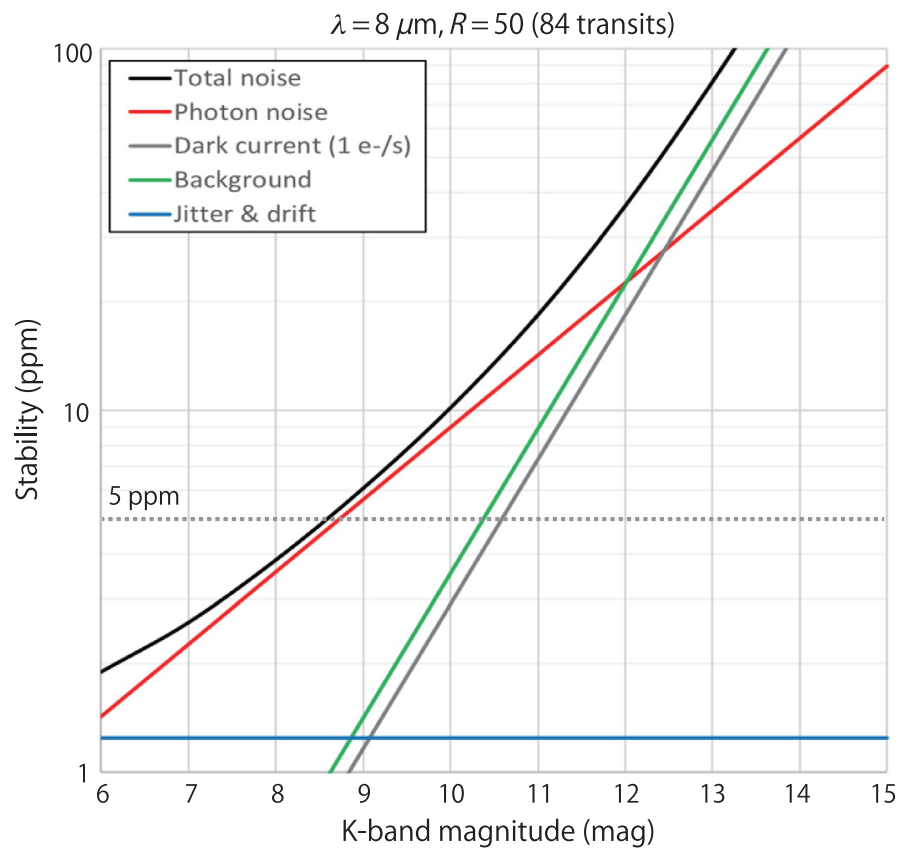

Fig. 11 Same as Fig. 9 but for at $8 \mu \mathrm{m}$ with $R=50$.

pointing correction function is provided by the MISC WFI module, the MISC-T in the upscope configuration does not include a Lyot-Coronagraph based TTS. Other than that, the optical design and the expected performance of the MICS-T are the same as those described in Sec. 2.

\subsection{MISC Wide Field Imager Science Traceability}

The imaging capability of the MISC WFI will be used for general science objectives. The spectroscopic capability with a resolving power of a few hundreds of the MISC WFI will 


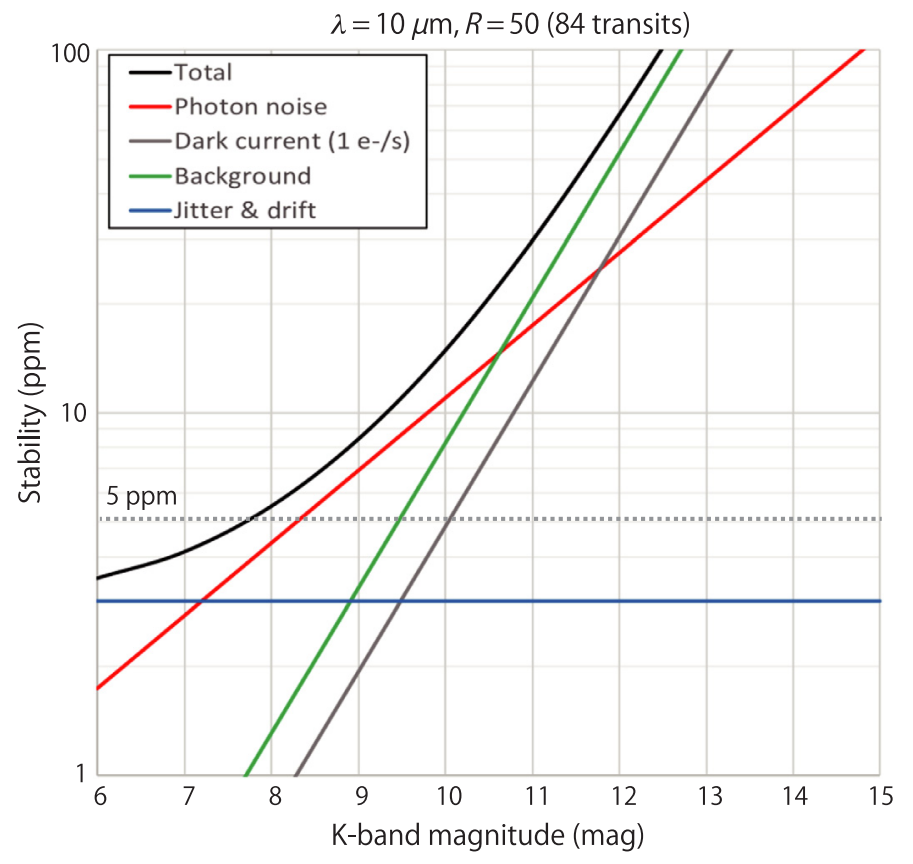

Fig. 12 Same as Fig. 9 but for at $10 \mu \mathrm{m}$ with $R=50$.

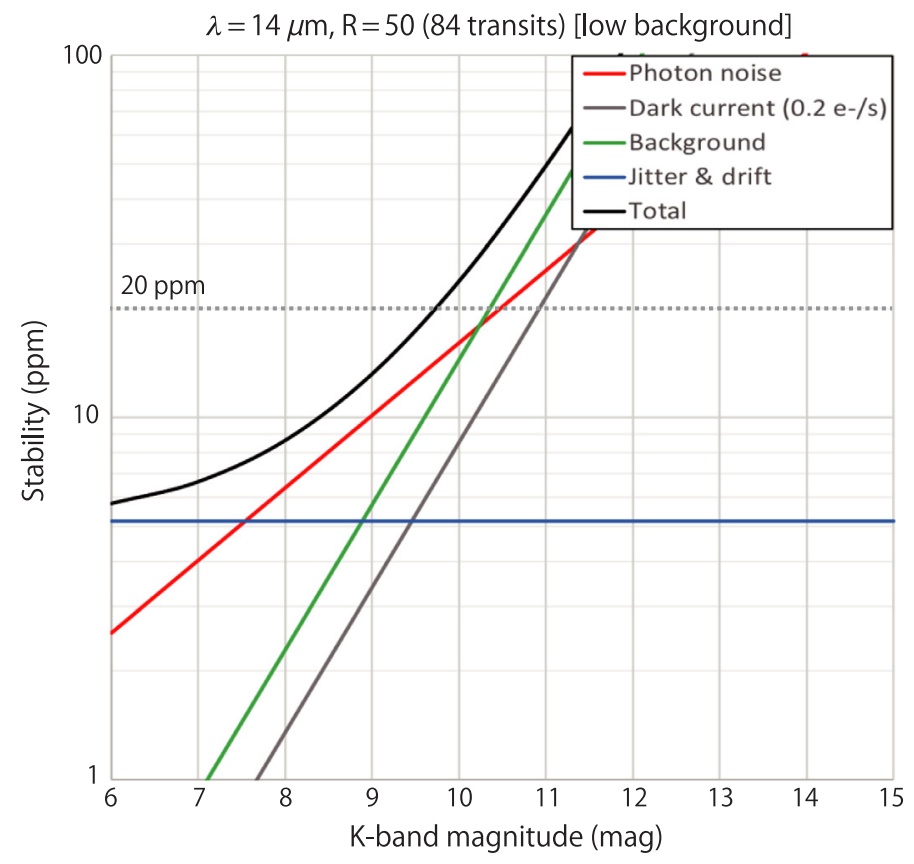

Fig. 13 Same as Fig. 9 but for at $14 \mu \mathrm{m}$ with $R=50$ (low background case ${ }^{10}$ ).

be used to measure the mid-infrared dust features and ionic lines at the redshift up to $z \sim 1$ in the Rise of Metals and Black Hole Feedback programs. ${ }^{4}$

\subsection{MISC WFI Instrument Description}

\subsubsection{MISC WFI operation principle}

The MISC WFI covers the wavelength from 5 to $28 \mu \mathrm{m}$ with two channels; WFI-short (WFI-S) covers the wavelength from 5 to $9 \mu \mathrm{m}$, and WFI-long (WFI-L) covers from 9 to $28 \mu \mathrm{m}$. 


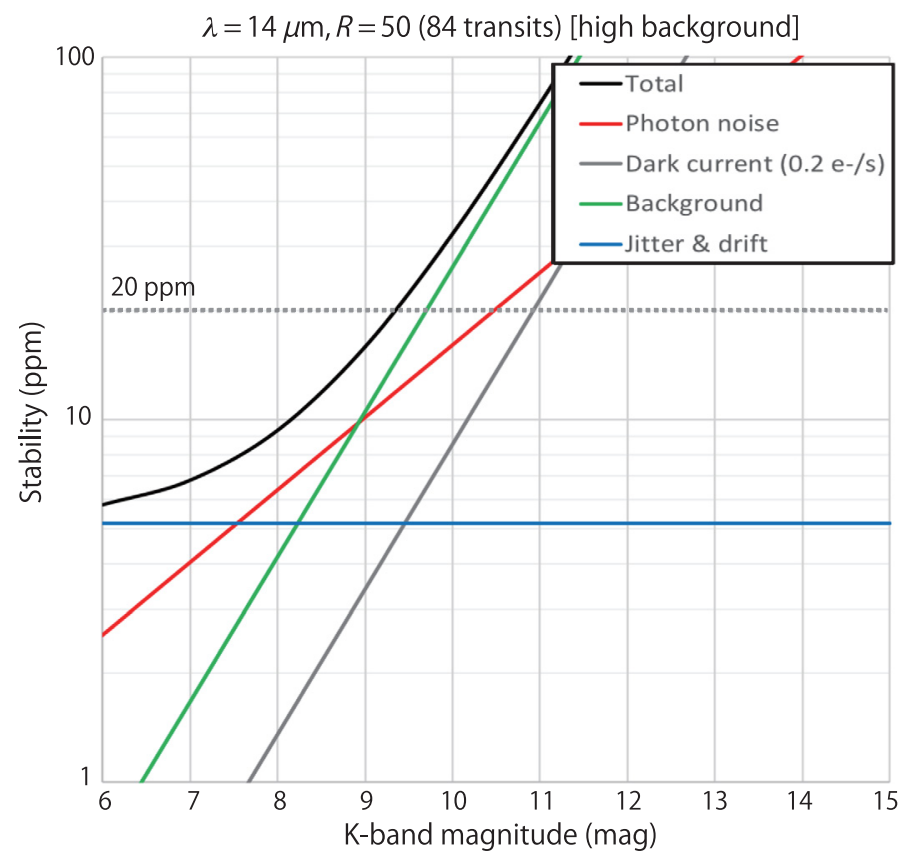

Fig. 14 Same as Fig. 9 but for at $14 \mu \mathrm{m}$ with $R=50$ (high background case ${ }^{11}$ ).

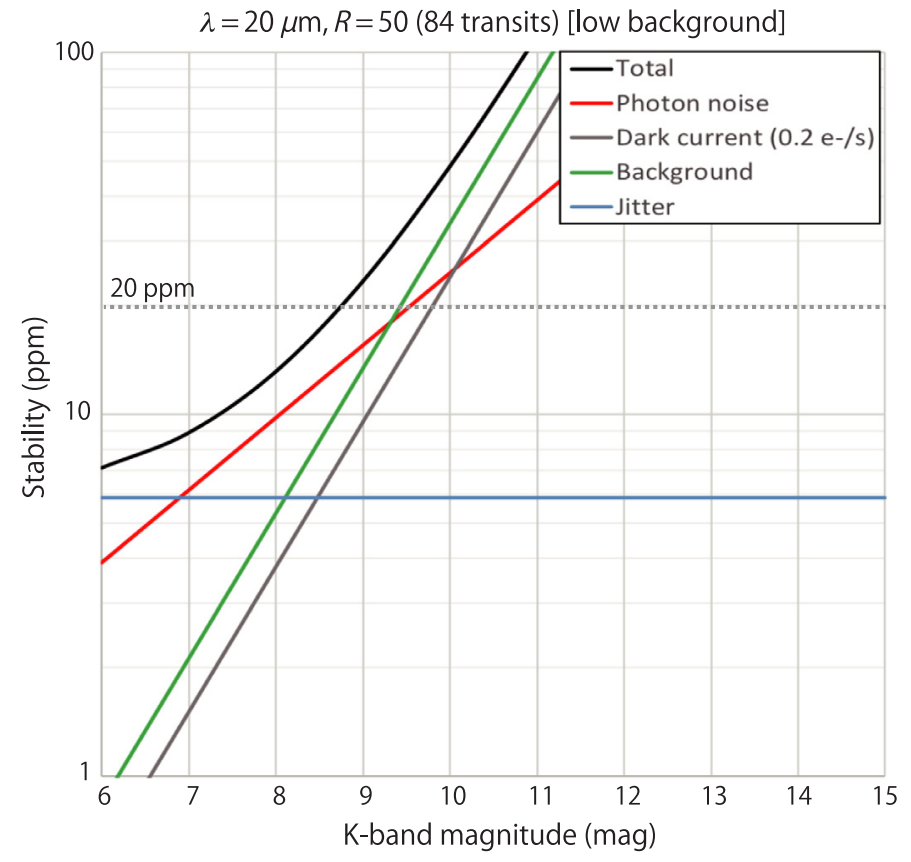

Fig. 15 Same as Fig. 9 but for at $20 \mu \mathrm{m}$ with $R=50$ (low background case ${ }^{10}$ ).

MISC WFI-S serves as the focal plane pointing and guiding for the observatory and has two redundant sets of $2 \mathrm{k} \times 2 \mathrm{k} \mathrm{Si}$ :As detector arrays. During the normal operation, one of the two Si:As detector arrays in the WFI-S is used, and the mirror changer switches the optical path if needed to use the other detector in case the performance of the first detector is degraded for some reason. Since the WFI-S array is also critically needed for the MISC-T performance, it is important that it is redundant. It should be noted that between the two channels, WFI-L channel was prioritized for science promise over WFI-S by the Science and Technology Definition Team (STDT), in case a trade choice would need to be made between the two. The block diagram of MISC WFI is shown in Fig. 17. 


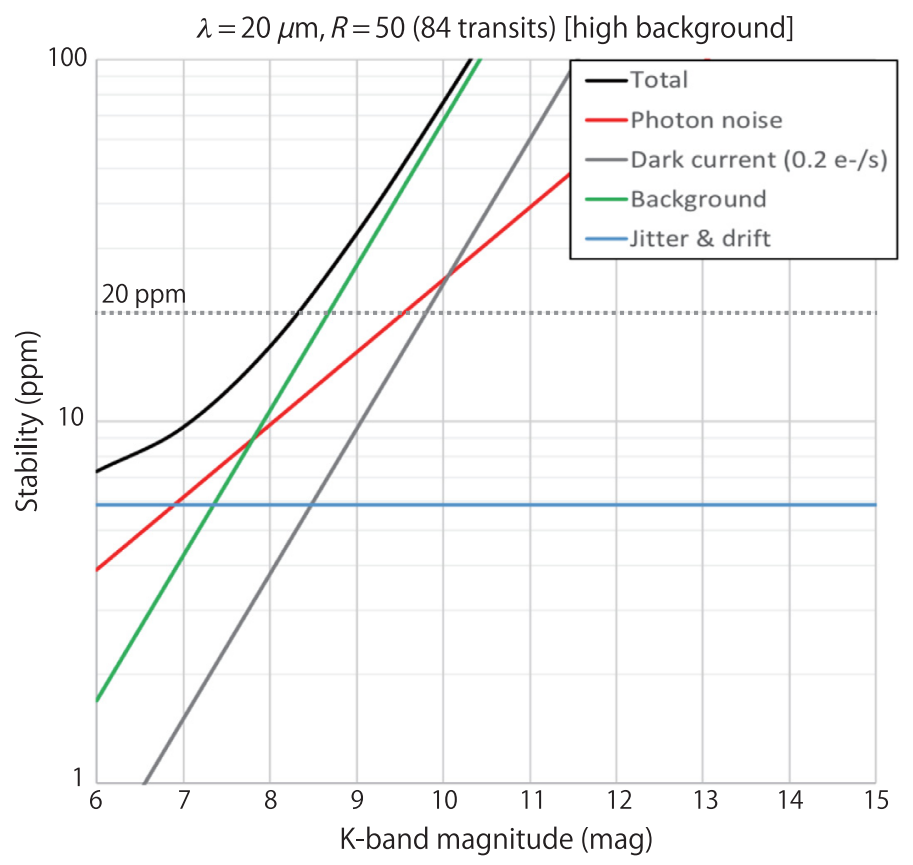

Fig. 16 Same as Fig. 9 but for at $20 \mu \mathrm{m}$ with $R=50$ (high background case ${ }^{11}$ ).

\subsubsection{MISC WFI optical design}

The MISC WFI offers a wide field imaging $\left(3^{\prime} \times 3^{\prime}\right)$ and low-resolution spectroscopic capability with filters and grisms covering from 5 to $28 \mu \mathrm{m}$ with a resolving power of $R \sim 300$. WFI-S and WFI-L share the same $3^{\prime} \times 3^{\prime}$ FOV by means of the beam splitter and the diffraction-limited image quality over the entire FOV is achieved at any wavelength from 5 to $28 \mu \mathrm{m}$ with the help of a deformable mirror (DM) and a tip tilt mirror in the fore optics of the MISC WFI. The reflected beam (i.e., 5 to $9 \mu \mathrm{m}$ ) by the beam splitter is diverted to WFI-S and the transmitted beam (i.e., 9 to $28 \mu \mathrm{m}$ ) is sent to WFI-L. The MISC WFI optical design includes a A) wave-front error correction system in the fore optics and B) the imager optics (see Fig. 18).

\subsubsection{MISC WFI detectors and read-out electronics}

The MISC WFI-S optical path contains two $2 \mathrm{k} \times 2 \mathrm{k}$ Si:As detector arrays and the MISC WFI$\mathrm{L}$ optical path contains a $2 \mathrm{k} \times 2 \mathrm{k} \mathrm{Si}$ :As detector array, each of which is bonded to a Si readout multiplexer operating at $\sim 8 \mathrm{~K}$ to provide good detective quantum efficiency in the 5 to $28 \mu \mathrm{m}$ wavelength range. This design has been used extensively in previous space missions such as Spitzer, AKARI, and JWST, and in the Stratospheric Observatory for Infrared Astronomy (SOFIA) instruments. The detector development work needed for the MISC WFI comprises a modest factor of two increase in the detector dimension format for the WFI module. ${ }^{8}$

Amplification of the signals from the detectors is a straightforward reuse of similar technology from previous space applications of Si:As BIB detectors such as Spitzer, JWST, and WISE, and will employ dedicated satellite chips that are located in close proximity to the detectors and also operate at cryogenic temperatures.

The readout electronics design of the MISC WFI detectors is very similar to that used in previous space missions and will incorporate flexible readout patterns and a variety of readout strategies including double-correlated and Fowler sampling technologies.

\subsubsection{MISC observing modes (upscope) and data rate}

MISC WFI offers six observing modes (i.e., guiding, imaging, low-resolution slit spectroscopy, low-resolution slit-less spectroscopy, and two MIR scan mapping modes) in addition to the MISC-T mode. (See Table 7.) 
Table 6 The MISC WFI instrument fact sheet.

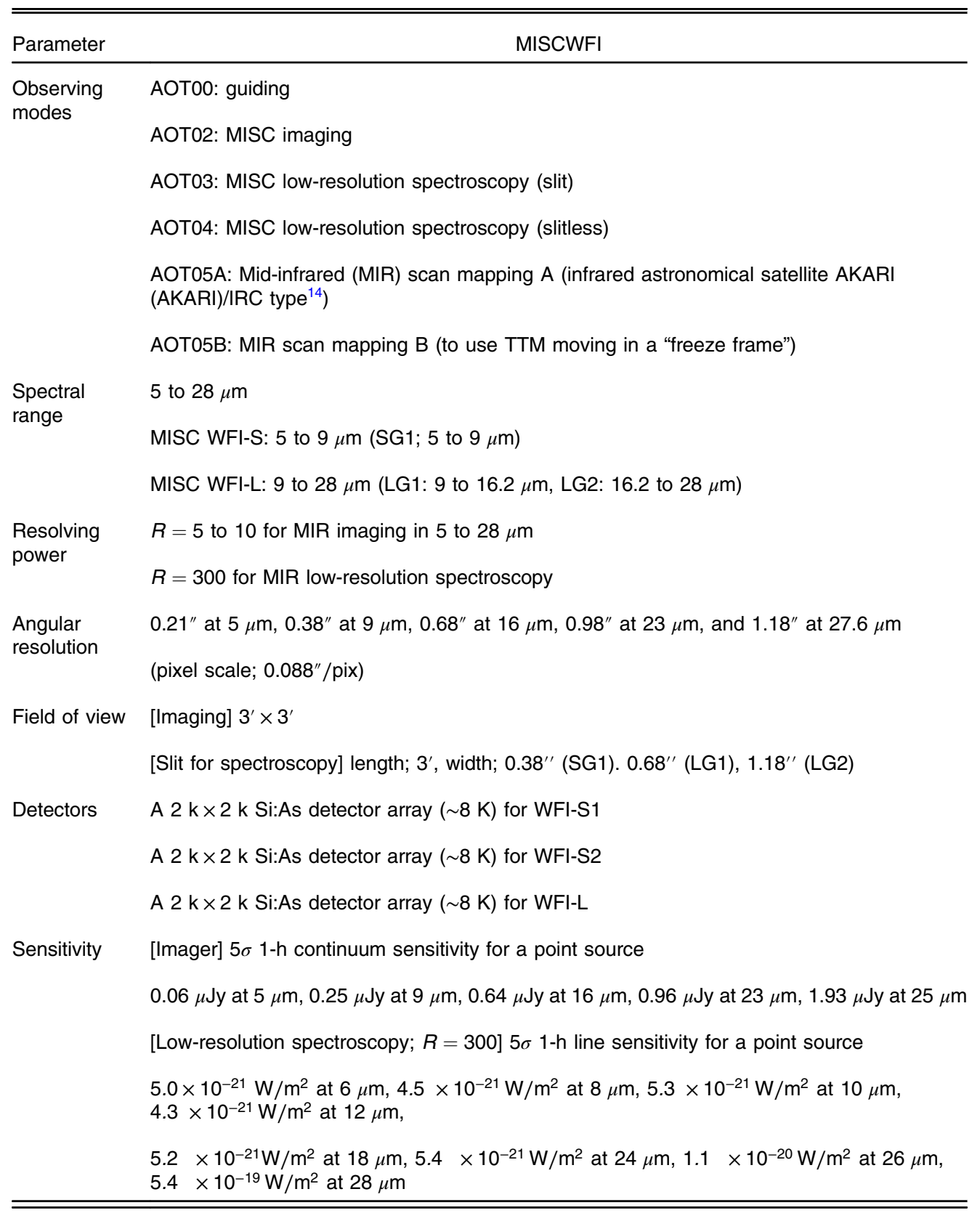

\subsubsection{MISC WFI thermal and mechanical design}

MISC WFI instrument components are located in two thermal zones. The instrument optics and detectors are located in a cold zone at the same $4.5 \mathrm{~K}$ temperature as the telescope. The detectors have a weak thermal link to this cold temperature so they can be heated slightly and held at a stable operating temperature. This operating temperature is $\sim 8 \mathrm{~K}$ for the Si:As detector. The drive electronics that handle instrument data collection and communication with the spacecraft are located in the $\sim 300 \mathrm{~K}$ warm zone.

The MISC WFI solid model is shown in Fig. 19. Table 8 lists the mass breakdown of cold component estimated each for beryllium and cordierite models. To reduce MISC WFI instrument mass, we assumed beryllium as the baseline material for the mirrors, mirror support structures, and base plate. 


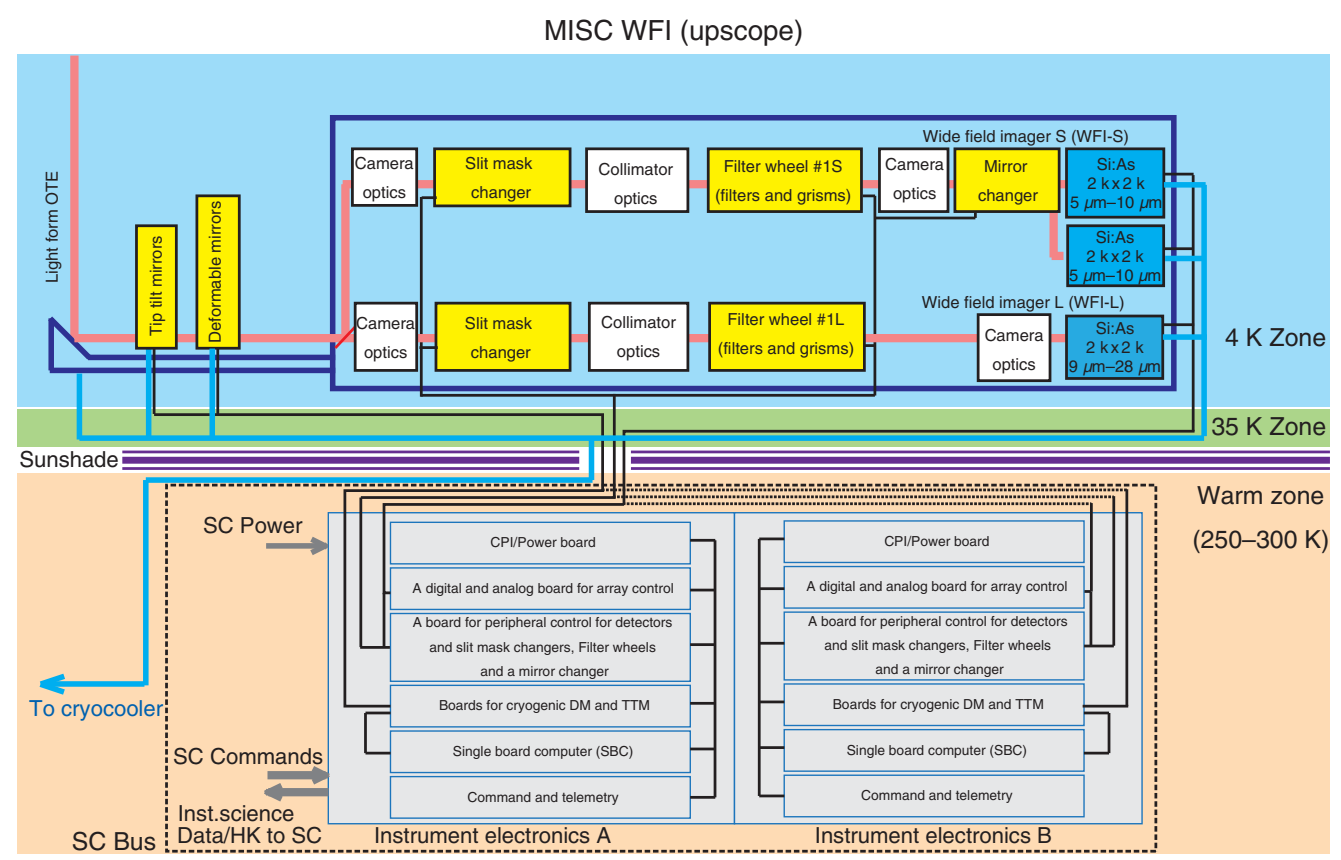

Fig. 17 The block diagram of the MISC WFI.

The MISC WFI module has the following mechanisms: (1) an DM assembly in the fore optics of the WFI module, (2) a tip-tilt mirror (TTM) assembly in the fore optics of the WFI module, (3) a slit mask changer in WFI-S, (4) a slit mask changer in WFI-L, (5) a filter wheel for WFI-S, (6) a filter wheel for WFI-L, and (7) a mirror changer in WFI-S. Since the telescope optics is limited to diffraction-limited image quality performance only at $30 \mu \mathrm{m}$ and longer wavelengths, the MISC WFI module needs to have its own internal wave-front error correction optics. The MISC WFI module includes a DM and a TTM in its fore-optics and achieves diffraction limited image performance at $>5 \mu \mathrm{m}$ for sources within a wide $3^{\prime} \times 3^{\prime}$ FOV. The slit mask changer used in MISC WFI-S has two 4-in. slots (a slit mask and a hole) and that in MISC WFI-L has three 4-in. slots (two slit masks and a hole). The filter wheel assembly used in each of the MISC WFI-S and WFI-L has triple wheels. Each wheel has six 3 -in. positions for band-pass filters and/or grisms for low-resolution spectroscopy. The mirror changer is placed just before the two Si:As detector arrays in the WFI-S to switches the optical path when needed to do so.

Table 9 lists the MISC WFI resource requirements (volume, mass, and power).

\subsubsection{Instrument control}

The MISCWFI has redundant dual-string warm electronics boxes (WEB A and WEB B for MISC WFI module). Each electronics box contains a SBC and four boards: (1) a CPI/ Power board, (2) a digital and analog board for array control (two $2 \mathrm{k} \times 2 \mathrm{k}$ Si:As arrays for WFI-S, one $2 \mathrm{k} \times 2 \mathrm{k} \mathrm{Si}$ :As array for WFI-L), (3) a board for peripheral control for detectors (two $2 \mathrm{k} \times 2 \mathrm{k}$ $\mathrm{Si}$ As arrays for WFI-S, one $2 \mathrm{k} \times 2 \mathrm{k}$ Si:As array for WFI-L), two slit mask wheels, two filter wheels, and one mirror changer, and (4) a control board for the cryogenic DM and TTM. A SBC is used to analyze the shape of the PSF and to give feedback to the DM and TTM to achieve the diffraction-limited image quality at $5 \mu \mathrm{m}$. This SBC also provides reference star centroid information that is sent to the Origins telescope pointing control.

\subsubsection{Risk management approach}

As an instrument on a NASA Class A mission, the MISC WFI module has a fully redundant, dual-string, cross-strapped design. This is particularly important for MISC WFI as the imaging 
MISC WFI-L

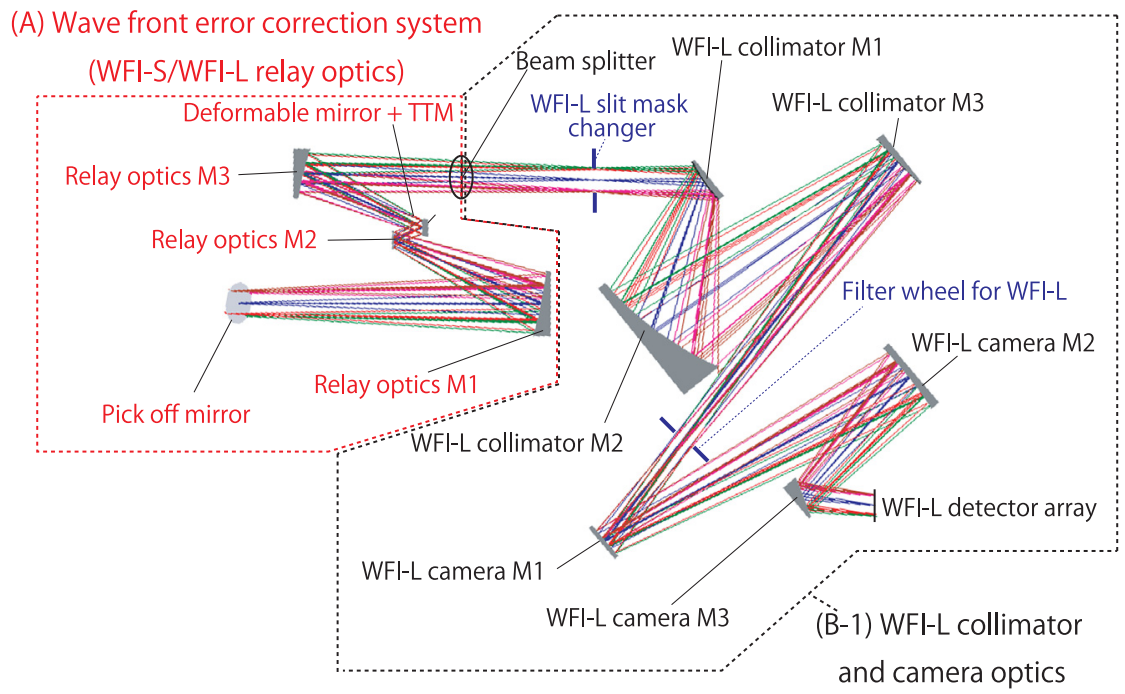

\section{MISC WFI-S}

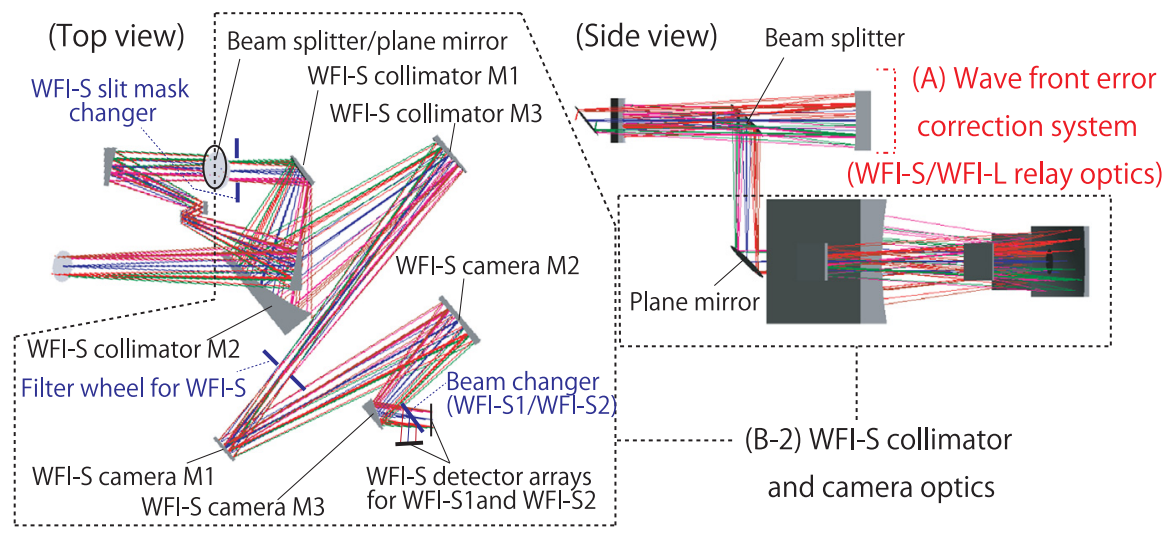

Fig. 18 The MISC WFI optical design.

array in the WFI-S may also serve as the focal plane pointing and guiding array for the observatory. Each of the MISC modules (i.e., MISC-T and MISC WFI) has its own warm electronics box each with two redundant sets of warm electronics. Within an electronics box the SBC and the rest of the warm electronics are cross-strapped as shown in Fig. 7. All of the moving mechanisms in the cold areas of MISC WFI have dual coils/actuators, with each coil/actuator capable of being driven by either of the A or B warm electronics sides. Although the focal plane arrays are not dual redundant, their multiplexer readouts are arranged so that sections of the arrays fail gracefully as was done for the JWST instrument arrays.

\subsubsection{MISC WFI alignment, integration, test, and calibration}

Since the MISC WFI module is used by the observatory for pointing, this instrument must meet confocality requirements with all other Origins instruments. As these other instruments all operate in the far-infrared, meeting this requirement should not be difficult. The twomodule configuration also greatly simplifies and speeds up instrument testing, as the modules can be tested independently and in parallel, if desired. Specialized test hardware development is required, but although the scope of this development will be significant, it is straightforward, with no new technology development required. The integration and test of the MISC instrument are expected to take 12 months, once all external test support hardware and software is ready. 
Table 7 MISC observing modes (upscope) and data rates.

\begin{tabular}{|c|c|c|}
\hline AOT & Mode & Data rate \\
\hline АОТО0 & Guiding & $2.63 \mathrm{Mbps}$ (max/average) ${ }^{\mathrm{a}}$ \\
\hline \multirow[t]{2}{*}{ AOT01 b } & MISC transit spectroscopy & $\begin{array}{l}\text { 3.73 Mbps }(\max )^{\mathrm{c}}-1.59 \mathrm{Mbps}(\text { average })^{\mathrm{d}} \\
\text { [MISC-T] }\end{array}$ \\
\hline & & $\begin{array}{l}2.63 \mathrm{Mbps} \text { (max/average) [Guiding with } \\
\text { MISC WFI-S] }\end{array}$ \\
\hline АОТ02 & MISC imaging & $\begin{array}{l}\text { 12.6 Mbps }(\max )^{\mathrm{e}}-0.45 \mathrm{Mbps}(\text { average })^{f} \\
{[\mathrm{MISC} \text { WFI-S and WFI-L] }}\end{array}$ \\
\hline АОТ03 & MISC low-resolution spectroscopy (slit) & $\begin{array}{l}\text { 12.6 Mbps }(\max )^{\mathrm{e}}-0.45 \mathrm{Mbps}(\text { average })^{f} \\
{[\mathrm{MISC} \text { WFI-S and WFI-L] }}\end{array}$ \\
\hline АОТ04 & MISC low-resolution spectroscopy (slit-less) & 12.6 $\operatorname{Mbps}(\max )^{\mathrm{e}}-4.5 \mathrm{Mbps}(\text { average })^{\mathrm{g}}[\mathrm{WFI}]$ \\
\hline АОТ05A & MIR scan mapping (AKARI/IRC type [14]) & 3.745 Mbps(max/average $)^{\mathrm{h}}$ \\
\hline АОТ05B & $\begin{array}{l}\text { MIR scan mapping(to use TTM moving in } \\
\text { a "freeze frame") }\end{array}$ & 16.78 Mbps(max/average) ${ }^{i}$ \\
\hline \multicolumn{3}{|c|}{$\begin{array}{l}\text { aUp to eight windows of } 32 \times 32 \text { pixels each of the MISC WFI-S module are read out at } 20 \mathrm{~Hz} \text {. } \\
\text { bSee also Table } 2 \text {. } \\
\text { cMax data rate of AOT01 is calculated for the shortest exposure } t_{\exp }=4 \mathrm{~s} \text {. } \\
{ }^{\mathrm{d}} \text { Average data rate is calculated for the longest exposure } t_{\exp }=10 \mathrm{~s} \\
\text { e Max data rate is calculated by assuming shortest exposure } t_{\exp }=2 \mathrm{~s} \text { and co-adding six exposures. } \\
{ }^{f} \text { Average data rate is calculated for the longest exposure time } t_{\exp }=300 \mathrm{~s} \text {. } \\
{ }^{9} \text { Average data rate is calculated for the longest exposure time } t_{\exp }=30 \mathrm{~s} \text {. } \\
\text { hUsing double lines }(2 \times 1 \text { pix } \times 2048 \text { pix) for the purpose of msec confirmation. Neighboring four lines are } \\
\text { read to avoid unstable behavior of the detector. Consequently } 2 \times(4+1+4)=18 \text { lines are read. Two lines } \\
\text { are down linked and other } 16 \text { lines are discarded. Assuming } 4 \mathrm{~s} \text { for full }(2048 \text { rows) readout, } 4 \times 18 / 2048= \\
35 \mathrm{~ms} \text { per double lines. The data rate becomes } 16 \text { bits } \times 2 \times 1 \text { pix } \times 2048 \text { pix } \times 2 \mathrm{ch} / 35 \mathrm{~ms}=3.745 \mathrm{Mbps} \text {. } \\
\text { 'Readout the half of the array at } 0.25 \mathrm{~Hz} \text {. The data rate becomes } 16 \text { bits } / \text { pix } \times 2048 \times 1024 \text { pix } \times 2 \mathrm{ch} \times \\
0.25 \mathrm{~Hz}=16.78 \mathrm{Mbps} \text {. }\end{array}$} \\
\hline
\end{tabular}

\subsubsection{MISC WFI predicted performance}

The following assumptions are made for the sensitivity estimate of the MISC WFI module (cf. Ref. 15). The 5 $\sigma$ 1-h line sensitivity of MISC WFI $(R=300)$ is shown in Fig. 20.

Exposure Time: The longest exposure time is determined as $300 \mathrm{~s}$ for the Si:As $2 \mathrm{k} \times 2 \mathrm{k}$ detectors (30 $\mu \mathrm{m}$ pix-1; Raytheon) taking account of the number of pixels affected by cosmic ray hit events during an exposure and assuming the cosmic ray hit event rate at L2 as $5 \times 10^{-4} \mathrm{~m}^{-2} \mathrm{~s}^{-1}$. $^{16}$

The Detector Performance: Detector Dark Current is assumed to be $0.06 \mathrm{e}^{-} \mathrm{s}^{-1}$ pixel $^{-1}$ for the Si:As $2 \mathrm{k} \times 2 \mathrm{k}$ detector. ${ }^{17}$ The readout noise (reduced up to $1 / 4$ by means of Flowler-16 sampling) is assumed to be $10 \mathrm{e}^{-}$for the Si:As $2 \mathrm{k} \times 2 \mathrm{k}$ detectors.

\section{MISC Heritage and Maturity}

The wavelength coverage and capabilities of MISC-T and MISC WFI partly overlap with those of JWST/mid-infrared instrument (MIRI) ${ }^{18}$ SOFIA instruments: Faint Object infrared Camera for the SOFIA Telescope ${ }^{19}$ Echeleon-Cross-Echelle Spectrograph, ${ }^{20}$ Space Infrared Telescope for Cosmology and Astrophysics (SPICA, a mission under study for ESA/JAXA) instrument studies: SPICA Mid-infrared Instrument (SMI, current), ${ }^{21}$ Mid-infrared Camera and Spectrometer, ${ }^{22}$ SPICA Coronagraph Instrument (SCI), ${ }^{23}$ Tokyo Atacama Observatory (TAO) Multi-field Imager for gazing at the UnKnown Universe (MIMIZUKU), ${ }^{24,25}$ Spitzer/IRS, ${ }^{26}$ and Thirty Meter Telescope Mid-Infrared Camera High-disperser \& IFU (MICHI), ${ }^{27}$ and NEOSM. ${ }^{9}$ Particularly, the long-wavelength $\mathrm{HgCdTe}$ detectors, which are employed in MISC-T-S and T-M, have been developed for the NEOSM (See details for Ref. 9). A summary of the MISC technology heritage is detailed in Table 10. 

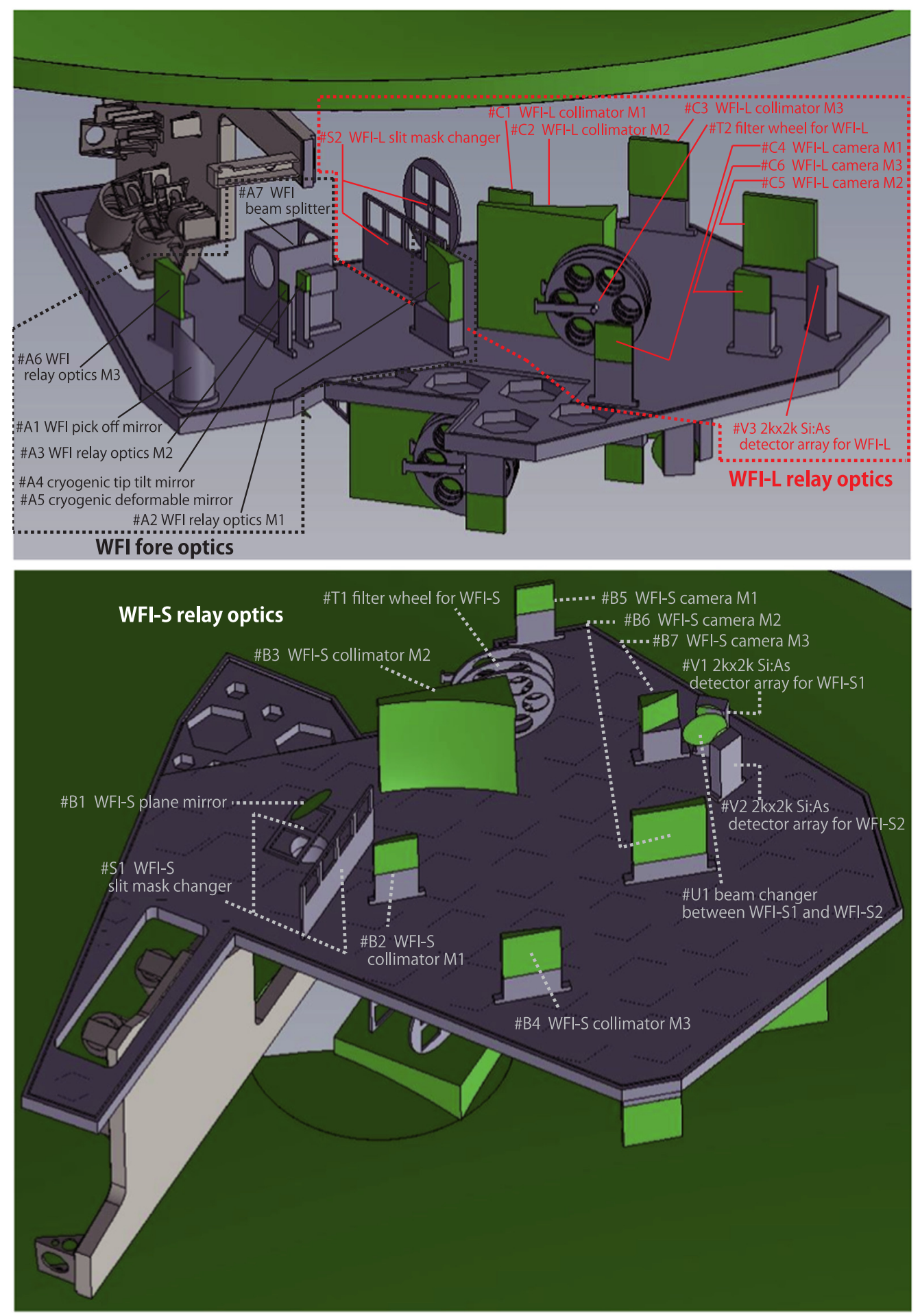

Fig. 19 A 3D solid model of MISC WFI module.

\section{MISC Enabling Technology}

Stable detectors are critical to MISC-T's overall performance. Detectors that meet the stability requirements do not exist today and development is necessary. Ref. 8 outlines the strategy to select detectors that meet requirements from three technologies: $\mathrm{HgCdTe}$ and $\mathrm{Si}$ :As detectors and TES bolometers. Onboard calibration sources to monitor and correct stability performance are also part of the development approach. 
Table 8 Mass breakdown of MISC WFI cold component.

\begin{tabular}{|c|c|c|c|}
\hline \multirow{2}{*}{\multicolumn{2}{|c|}{ Subassembly/part name }} & \multicolumn{2}{|c|}{ Mass $(\mathrm{kg})$} \\
\hline & & \multirow{2}{*}{$\frac{\text { Beryllium model }^{\mathrm{a}}}{0.18}$} & \multirow{2}{*}{$\frac{\mathrm{CO} 20 \text { model }^{\mathrm{b}}}{0.30}$} \\
\hline WFI fore optics and & \#A1 WFI pick off mirror & & \\
\hline & \#A2 WFI relay optics M1 & 0.41 & 0.68 \\
\hline & \#A3 WFI relay optics M2 & 0.02 & 0.04 \\
\hline & \#A4 cryogenic TT mirror & 1.00 & 1.00 \\
\hline & \#A5 cryogenic Deformable Mirror & 6.00 & 6.00 \\
\hline & \#A6 WFI relay optics M3 & 0.20 & 0.34 \\
\hline & \#A7 WFI beam splitter & 1.00 & 1.00 \\
\hline \multirow{7}{*}{$\begin{array}{l}\text { WFI-S optics and } \\
\text { mirror supports }\end{array}$} & \#B1 plane mirror & 0.20 & 0.30 \\
\hline & \#B2 WFI-S collimator M1 & 0.60 & 1.00 \\
\hline & \#B3 WFI-S collimator M2 & 3.86 & 6.43 \\
\hline & \#B4 WFI-S collimator M3 & 1.40 & 2.34 \\
\hline & \#B5 WFI-S camera M1 & 0.30 & 0.50 \\
\hline & \#B6 WFI-S camera M2 & 1.34 & 2.24 \\
\hline & \#B7 WFI-S camera M3 & 3.41 & 5.69 \\
\hline \multirow{6}{*}{$\begin{array}{l}\text { WFI-L optics and } \\
\text { mirror supports }\end{array}$} & \#C1 WFI-L collimator M1 & 0.60 & 1.00 \\
\hline & \#C2 WFI-L collimator M2 & 3.86 & 6.4 \\
\hline & \#C3 WFI-L collimator M3 & 1.40 & 2.34 \\
\hline & \#C4 WFI-L camera M1 & 0.30 & 0.50 \\
\hline & \#C5 WFI-L camera M2 & 1.34 & 2.24 \\
\hline & \#C6 WFI-L camera M3 & 3.41 & 5.69 \\
\hline \multicolumn{2}{|c|}{ \#S1 WFI-S slit mask changer } & 3.80 & 3.80 \\
\hline \multicolumn{2}{|l|}{ \#T1 WFI-S filter wheel } & 5.00 & 5.00 \\
\hline \multicolumn{2}{|c|}{ \#U1 beam changer between WFI-S1 and WFI-S2 } & 2.00 & 2.00 \\
\hline \multicolumn{2}{|c|}{ \#V1 WFI-S1 detector unit (including Box) } & 1.36 & 1.36 \\
\hline \multicolumn{2}{|c|}{ \#V2 WFI-S2 detector unit (including Box) } & 1.36 & 1.36 \\
\hline \multicolumn{2}{|c|}{ \#S2 WFI-L slit mask changer } & 3.80 & 3.80 \\
\hline \multicolumn{2}{|l|}{ \#T2 WFI-L filter wheel } & 5.00 & 5.00 \\
\hline \multicolumn{2}{|c|}{ \#V3 WFI-L detector unit (including box) } & 1.36 & 1.36 \\
\hline \multicolumn{2}{|c|}{ Instrument optical bench for WFI } & 66.00 & 110.0 \\
\hline \multicolumn{2}{|l|}{ Cover } & 6.00 & 6.00 \\
\hline \multicolumn{2}{|l|}{ Total } & 126.51 & 185.71 \\
\hline
\end{tabular}

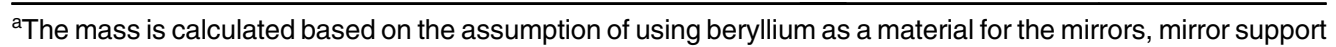
structures, and the base plate.

${ }^{\mathrm{b}}$ The mass is calculated based on the assumption of using Cordierite CO720 as a material for the mirrors, mirror support structures, and the base plate. 
Table 9 MISC WFI module resource requirements.

\begin{tabular}{|c|c|c|c|}
\hline \multirow[b]{2}{*}{ Instrument resource type } & \multicolumn{2}{|l|}{ Resource and units } & \multirow[b]{2}{*}{ MISC WFI (upscope) } \\
\hline & Resource & Units & \\
\hline $\begin{array}{l}\text { Information related to } \\
\text { the mechanical model }\end{array}$ & Volume (cold component) & $m^{3}(m \times m \times m)$ & $3.23(1.7 \times 1.9 \times 1.0)$ \\
\hline \multirow{12}{*}{$\begin{array}{l}\text { Information related to } \\
\text { the thermal model }\end{array}$} & Mass (cold component) & $\mathrm{kg}$ & $126.51^{1}$ \\
\hline & Mass (warm component) & $\mathrm{kg}$ & 16.00 \\
\hline & Mass (Harnessing and others) & $\mathrm{kg}$ & 27.70 \\
\hline & Total mass & $\mathrm{kg}$ & 170.21 \\
\hline & Total peak power (cold part) & W & 0.197 \\
\hline & Total peak power (warm part) & W & 120 \\
\hline & Total average power (cold part) & W & 0.050 \\
\hline & Total average power (warm part) & W & 46 \\
\hline & Total standby power (cold part) & W & 0.009 \\
\hline & Total standby power (warm part) & W & 46 \\
\hline & Average power dissipation (detectors) & W & 0.008 \\
\hline & Average power dissipation (heater) & W & 0.009 \\
\hline
\end{tabular}

Note 1: The mass is calculated based on the assumption of using beryllium as a baseline material for the mirrors, mirror support structures, and the base plate.

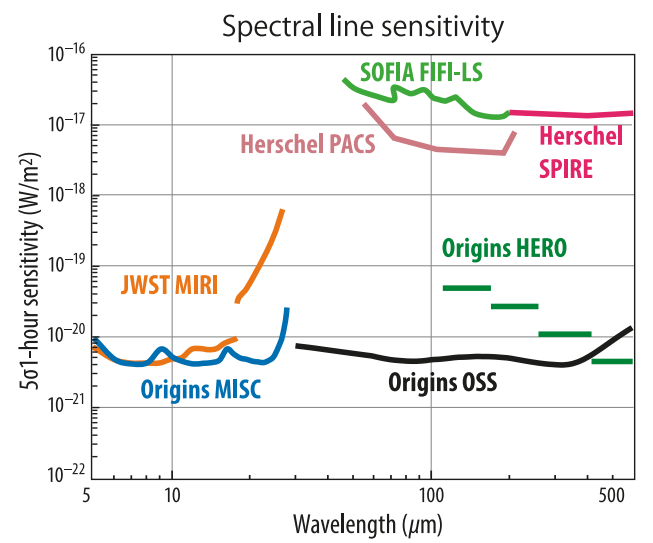

Fig. $205 \sigma$ 1-h line sensitivity of OST/MISC WFI $(R=300)$.

In addition, there are two new technologies that will need to be further developed to include the MISC upscope capabilities: larger format $\mathrm{Si}$ :As arrays and a DM capable of operation at $\sim 8 \mathrm{~K}$ temperatures. The increase in Si:As array format size is expected to be relatively straightforward, since as other than the array size the current performance of the detectors used in the JWST MIRI instrument will be sufficient for the MISC WFI. A cryogenic DM has been demonstrated (e.g., Refs. 23 and 30), although not at quite as low as the temperature of the MISC WFI.

\section{Summary}

The MISC instrument studied for the Origins had two major components: a transit spectrometer (MISC-T) and a camera with low-resolution grism spectroscopy (MISC WFI). 
Table 10 MISC component heritage.

\begin{tabular}{|c|c|c|}
\hline Description & Subsystem/component & Heritage \\
\hline Densified pupil spectrometer & Subsystem & New technology $y^{5,6}$ \\
\hline Be mirrors and structures (including base plate) & Component & NIRCam instrument on $\mathrm{JWST}^{28}$ \\
\hline $2 \mathrm{k} \times 2 \mathrm{k} \mathrm{Si}$ As with a calibration source & Component & JWST/MIRI, ${ }^{18}$ SPICA/SMI ${ }^{21}$ \\
\hline $2 \mathrm{k} \times 2 \mathrm{k} \mathrm{HgCdTe}$ & Component & NEOSM $^{9}$ \\
\hline $\begin{array}{l}\text { Beam splitter, short-wave cut filters } \\
\text { (multi-layer interference filters) }\end{array}$ & Component & AKARI/IRC, ${ }^{29}$ SPICA/MCS ${ }^{22}$ \\
\hline Lyot Stop (for TTS) & Component & $\mathrm{SPICA} / \mathrm{SCl}^{23}$ and others \\
\hline \multirow[t]{3}{*}{ Cryogenic DM } & Component & $\mathrm{SPICA} / \mathrm{SCl}^{23,30}$ \\
\hline & & $\begin{array}{l}\text { AO technology for Subaru } \\
\text { telescope and } \mathrm{TMT}^{31,32}\end{array}$ \\
\hline & & Cryogenic DM studied in SRON \\
\hline Cryogenic TTM & Component & $\begin{array}{l}\text { SPICA/SCI, }{ }^{23} \text { JWST/NIRCam, }{ }^{28} \\
\text { and TAO/MIMIZUKU }{ }^{24,25}\end{array}$ \\
\hline
\end{tabular}

MISC-T module has been studied as the MISC baseline and is optimized for measurements of bio-signatures in the atmospheres of transiting exoplanets. MISC-T employs a densified pupil spectrometer design and provides spectroscopy simultaneously over 2.8 to $20 \mu \mathrm{m}$ with exquisite stability and precision ( $<5 \mathrm{ppm}$ from 2.8 to $10 \mu \mathrm{m},<20 \mathrm{ppm}$ from 10 to $20 \mu \mathrm{m}$ ). With these capabilities, MISC-T makes the Origins a powerful tool to bring a revolutionary progress, in particular, in exoplanet sciences.

MISC WFI has been studied as a second module for a MISC upscope and offers a wide field imaging $\left(3^{\prime} \times 3^{\prime}\right)$ and low-resolution spectroscopic capability with filters and grisms for 5 to $28 \mu \mathrm{m}$. Combined with the spectroscopic capability in the FIR provided particularly by the Origins Survey Spectrometer (OSS), ${ }^{33}$ the MISC WFI widens the wavelength coverage of Origins down to $5 \mu \mathrm{m}$, which makes the Origins a powerful tool to diagnose the physical and chemical condition of the ISM using dust features, molecules lines and atomic and ionic lines. The MISC WFI provides Origins with a focal plane guiding function for the other Origins science instruments including OSS, Far-infrared Imager and Polarimeter, ${ }^{34}$ Origins HEterodyne Receiver for Origins ${ }^{35}$ and its own use.

\section{Acknowledgments}

To enable the community to prepare for the 2020 Decadal Survey, NASA sponsored studies of four large mission concepts, of which Origins was one. The authors thank all the members of the Origins STDT and the engineering study team for their continuous support. The authors thank H. Shibai, T. Onaka, T. Nakagawa, H. Matsuhara, and T. Yamada for providing useful advice. This work is supported in part by a Grant-in-Aid for Young Scientists (A) (Grant No. 16H05997) and a Grant-in-Aid for Scientific Research (C) (Grant No. 19K03909). This work is also financially supported in part by Promoted Research for International Joint Mission by Advisory committee for Space science and engineering, JAXA.

\section{References}

1. D. Leisawitz et al., "The Origins Space Telescope: baseline mission concept," J. Astron. Telesc. Instrum. Syst. 7(1), 011002 (2021).

2. I. Sakon et al., "The mid-infrared imager spectrometer, coronagraph (MISC) for the Origins Space Telescope (OST)," Proc. SPIE 10698, 1069817 (2018). 
3. C. Battersby et al., "The origins space telescope," Nat. Astron. 2, 596-599 (2018).

4. M. Meixner et al., "Origins space telescope science to design traceability," J. Astron. Telesc. Instrum. Syst. 7(1), 011012 (2021).

5. T. Matsuo et al., "A new concept for spectrophotometry of exoplanets with space-borne telescopes," Astrophys. J. 823, 139 (2016).

6. T. Matsuo et al., "A highly stable spectrophotometric capability for the Origins Space Telescope (OST) mid-infrared imager, spectrometer, coronagraph (MISC)," Proc. SPIE 10698, 1069844 (2018).

7. J. Corsetti et al., "Optical design of the origins space telescope," J. Astron. Telesc. Instrum. Syst. 7(1), 011010 (2021).

8. T. L. Roellig et al., "Mid-infrared detector development for the origins space telescope," J. Astron. Telesc. Instrum. Syst. 6(4), 041503 (2020).

9. M. L. Dorn et al., "Proton irradiation results for long-wave $\mathrm{HgCdTe}$ infrared detector arrays for Near-Earth Object Camera," J. Astron. Telesc. Instrum. Syst. 2, 036002 (2016).

10. A. Glasse et al., "The mid-infrared instrument for the James Webb Space Telescope, IX: predicted sensitivity," Publ. Astron. Soc. Pac. 127, 686 (2015).

11. E. L. Wright, "Angular power spectra of the COBE DIRBE maps," Astrophys. J. 496, 1 (1998).

12. P. J. Love, " $1024 \times 1024$ Si: as IBC detector arrays for MID-IR astronomy," Proc. SPIE 6276, 62761Y (2006).

13. S. Goda and T. Matsuo, "A new method for calibration of gain variation in a detector system," Astron. J. 156, 288 (2018).

14. D. Ishihara et al., "The AKARI/IRC mid-infrared all-sky survey," Astron. Astrophys. 514, 1 (2010).

15. I. Sakon et al., "Sensitivity estimates for the SPICA Mid-Infrared Instrument (SMI)," Proc. SPIE 9904, 99043V (2016).

16. B. M. Swinyard et al., "Sensitivity estimates for the mid-infrared instrument (MIRI) on the JWST," Proc. SPIE 5487, 785-793 (2004).

17. M. E. Ressler et al., "Performance of the JWST/MIRI Si:As detectors," Proc. SPIE 7021, 702100 (2008).

18. G. H. Rieke et al., "The mid-infrared instrument for the James Webb Space Telescope, I: Introduction," Publ. Astron. Soc. Pac. 127, 584-594 (2015).

19. T. L. Herter et al., "FORCAST: a mid-infrared camera for SOFIA," J. Astron. Instrum. 7, 1840005-451 (2018).

20. M. J. Richter et al., "EXES: The Echelon-cross-Echelle Spectrograph for SOFIA,” J. Astron. Instrum. 7, 1840013 (2018).

21. H. Kaneda et al., "SPICA mid-infrared instrument (SMI): conceptual design and feasibility studies," Proc. SPIE 10698, 106980C (2018).

22. H. Kataza et al., "Mid-infrared camera and spectrometer on board SPICA," Proc. SPIE 8442, 84420Q (2012).

23. K. Enya et al., "A high dynamic-range instrument for SPICA for coronagraphic observation of exoplanets and monitoring of transiting exoplanets," Proc. SPIE 8146, 81460Q (2011).

24. T. Miyata et al., "Development of a new mid-infrared instrument for the TAO 6.5-m telescope," Proc. SPIE 7735, 77353P (2010).

25. T. Kamizuka et al., "Laboratory performance evaluation of the mid-infrared camera and spectrograph MIMIZUKU for the TAO 6.5-m telescope," Proc. SPIE 10702, 107022H (2018).

26. J. R. Houck et al., "The infrared spectrograph (IRS) on the Spitzer Space Telescope," Astrophys. J. 154, 18-24 (2004).

27. C. Packham et al., "The key science drivers for MICHI: a thermal-infrared instrument for the TMT," Proc. SPIE 10702, 10702A0 (2018).

28. M. J. Rieke et al., "Overview of James Webb Space Telescope and NIRCam's role," Proc. SPIE 5904, 1-8 (2005).

29. T. Onaka et al., "The infared camera (IRC) for AKARI—design and imaging performance," Publ. Astron. Soc. Jpn. 59S, 401 (2007). 
30. A. Takahashi et al., "Laboratory demonstration of a cryogenic deformable mirror for wavefront correction of space-borne infrared telescopes," Appl. Opt. 56, 6694 (2017).

31. S. Oya et al., "Deformable mirror design of Subaru/LGSAO system," Proc. SPIE 5490, 1546-1555 (2004).

32. S. Oya et al., "Characterization of vibrating shape of a bimorph deformable mirror," Proc. SPIE 7015, 70153R (2008).

33. M. Bradford et al., "The Origins Survey Spectrometer (OSS): revealing the hearts of distant galaxies and forming planetary systems with ultrasensitive far-IR spectroscopy," J. Astron. Telesc. Instrum. Syst. 7(1), (2021).

34. J. Staguhn et al., "Far-infrared imager and polarimeter (FIP)," J. Astron. Telesc. Instrum. Syst. 7(1), (2021).

35. M. Wiedner et al., "Heterodyne receiver for origins (HERO)," J. Astron. Telesc. Instrum. Syst. 7(1), 011007 (2021).

Itsuki Sakon is an assistant professor at the University of Tokyo. He received his BS and MS degrees in the University of Tokyo of Astronomy in 2003 and 2005, respectively, and his PhD degree in science from the University of Tokyo in 2008. His current research interests include mid-infrared astronomical instrumentation development and infrared observational astronomy, laboratory astrophysics and astrochemistry. He has conducted research and published papers on observational and experimental studies of interstellar dust and organics and in infrared instrument development.

Thomas L. Roellig has worked in the NASA civil service as an astrophysicist at the NASA Ames Research Center since 1980 and is currently the chief of the Astrophysics Branch at Ames. His scientific research interests have spanned a wide range of infrared astronomy and astronomical instrumentation development. He has conducted research and published papers in infrared instrument development, solar science, solar system science, star formation, interstellar medium, and brown dwarf astronomy.

Kimberly Ennico-Smith has built infrared cameras, spectrometers, suborbital instruments, and lunar payloads. She has tested detectors at particle accelerators. She has served as SOFIA Project Scientist and New Horizons deputy Project Scientist. She has been a STDT member for Origins Space Telescope, authored 120+ peer-reviewed papers and delivered 50+ invited technical talks and $70+$ public presentations. Asteroid 154587 Ennico is named for her. She is currently the VIPER lunar rover deputy project scientist.

Taro Matsuo is an associate professor of physics at Nagoya University. His research is focused on the field of exoplanet science, with an emphasis on developing several instrument concepts for characterization of nearby habitable planet candidates, including transit spectrographs, interferometers, and coronagraphs.

Biographies of the other authors are not available. 